\title{
On primitives and conjugate harmonic pairs in Hermitean Clifford analysis
}

\author{
F. Brackx* H. De Schepper*, R. Lávička ${ }^{\ddagger} \&$ V. Souček ${ }^{\ddagger}$ \\ * Clifford Research Group, Department of Mathematical Analysis, \\ Faculty of Engineering and Architecture, Ghent University \\ Building S22, Galglaan 2, B-9000 Gent, Belgium \\ $¥$ Mathematical Institute, Faculty of Mathematics and Physics, Charles University \\ Sokolovská 83, 18675 Praha, Czech Republic
}

\begin{abstract}
The notion of a conjugate harmonic pair in the context of Hermitean Clifford analysis is introduced as a pair of specific harmonic functions summing up to a Hermitean monogenic function in an open region $\Omega$ of $\mathbb{C}^{n}$. Under certain geometric conditions on $\Omega$ the conjugate harmonic to a given specific harmonic is explicitly constructed and the potential or primitive of a Hermitean monogenic function is determined.
\end{abstract}

MSC Classification: 30G35

Key words: conjugate harmonics, monogenic primitive, Clifford analysis

\section{Introduction}

When considering a region, i.e. an open connected set $\Omega$ of the complex plane, it is well-known, by the Hauptsatz of the Cauchy theory (see e.g. [15]), that the following are equivalent:

(i) $\Omega$ is simply connected;

(ii) every holomorphic function in $\Omega$ has a holomorphic primitive $F$, such that $\partial_{z} F=f$ in $\Omega$, with $\partial_{z}=\frac{1}{2}\left(\partial_{x}-i \partial_{y}\right)$ the conjugate Cauchy-Riemann operator;

(iii) every harmonic function $u$ in $\Omega$ has a conjugate harmonic $v$ such that $u+i v$ is holomorphic in $\Omega$.

Note that if $F=U+i V$ is a holomorphic primitive of the holomorphic function $f$ in $\Omega$, then automatically $2 U$ and $2 i V$ are two conjugate harmonic primitives of $f$ in $\Omega$ since

$$
\begin{aligned}
f & =\partial_{z} F=\partial_{x} F=-i \partial_{y} F \\
& =\partial_{x} U+i \partial_{x} V=-i \partial_{y} U+\partial_{y} V=\left(\partial_{x}-i \partial_{y}\right) U=\left(\partial_{x}-i \partial_{y}\right)(i V) \\
& =\partial_{z}(2 U)=\partial_{z}(2 i V)
\end{aligned}
$$

Also note that if $u$ and $v$ are conjugate harmonics in $\Omega$ and $F=U+i V$ is a holomorphic primitive of the holomorphic function $f=u+i v$ in $\Omega$, then

$$
u=\partial_{x} U=\partial_{y} V \text { and } v=\partial_{x} V=-\partial_{y} U
$$

Even when the region $\Omega$ is not simply connected, it is still possible to construct locally a harmonic primitive $\widetilde{F}$ of a given holomorphic function $f$ in $\Omega$. Indeed there holds the following result involving the conjugate Pompeiu transform, the Pompeiu transform itself being an inverse of the CauchyRiemann operator. 
Lemma 1. Let $f$ be holomorphic in the region $\Omega$. For each $\Omega_{0} \subset \Omega$ such that $\overline{\Omega_{0}}$ is compact and contained in $\Omega$, the function $\widetilde{F}$ given by

$$
\widetilde{F}(z)=\frac{1}{2 \pi i} \int_{\Omega_{0}} \frac{f(w)}{w-\bar{z}} d w \wedge d \bar{w}
$$

is a harmonic primitive of $f$ in $\Omega_{0}$, satisfying there $\partial_{z} \widetilde{F}=f$ and $\Delta \widetilde{F}=0$.

As an example consider the holomorphic function $\frac{1}{z}$ in the region $\Omega=\mathbb{C} \backslash\{0\}$ which has no holomorphic primitive in $\Omega$, but which shows there the harmonic primitive $\widetilde{F}=2 \ln |\underline{z}|=\ln \left(x^{2}+y^{2}\right)$.

In [29] the notion of a conjugate harmonic pair was generalized to Euclidean space $\mathbb{R}^{m+1}$ as follows: a real vector field $F^{*}=\left[U_{0}, U_{1}, \ldots, U_{m}\right]$ is called an $(m+1)$-tuple of conjugate harmonics in a region $\Omega$ of $\mathbb{R}^{m+1}$ if it satisfies in $\Omega$ the so-called Riesz system

$$
\begin{cases}\operatorname{div} F^{*} & =0 \\ \operatorname{curl} F^{*} & =0\end{cases}
$$

or more explicitly

$$
\left\{\begin{array}{l}
\partial_{X_{0}} U_{0}+\partial_{X_{1}} U_{1}+\ldots+\partial_{X_{m}} U_{m}=0 \\
\partial_{X_{i}} U_{j}-\partial_{X_{j}} U_{i}=0, i \neq j, i, j=0, \ldots, m
\end{array}\right.
$$

If $\Omega \subset \mathbb{R}^{m+1}$ is moreover contractible to a point, then such an $(m+1)$-tuple $F^{*}$ of conjugate harmonics may be realized as the gradient of a real-valued harmonic function $\phi$ in $\Omega: F^{*}=\vec{\nabla} \phi$ or $U_{j}=\partial_{X_{j}} \phi, j=0, \ldots, m$.

In [5] an alternative notion of conjugate harmonics in Euclidean space was introduced within the context of so-called Clifford analysis. Clifford analysis, in its most basic form, is a higher dimensional generalization of holomorphic function theory in the complex plane, and a refinement of harmonic analysis, see e.g. $[5,21,17,23,22]$. At the heart of this function theory lies the notion of a monogenic function, i.e. a Clifford algebra-valued null solution of the Dirac operator $\underline{\partial}=\sum_{\alpha=1}^{m} e_{\alpha} \partial_{X_{\alpha}}$, where $\left(e_{1}, \ldots, e_{m}\right)$ is an orthonormal basis of $\mathbb{R}^{m}$ underlying the construction of the real Clifford algebra $\mathbb{R}_{0, m}$ of signature $(0, m)$. We refer to this setting as the Euclidean case, since the fundamental group leaving the Dirac operator $\underline{\partial}$ invariant is the orthogonal group $\mathrm{O}(m ; \mathbb{R})$, which is doubly covered by the $\operatorname{Pin}(m)$ group of the Clifford algebra. Similarly, the Clifford algebra $\mathbb{R}_{0, m+1}$ is generated by the basis $\left(e_{0}, e_{1}, \ldots, e_{m}\right)$ and, by singling out the basis vector $e_{0}$, it can be split as

$$
\mathbb{R}_{0, m+1}=\mathbb{R}_{0, m} \oplus \overline{e_{0}} \mathbb{R}_{0, m}
$$

Accordingly, a function $f$ defined on $\mathbb{R}^{m+1}$ with values in $\mathbb{R}_{0, m+1}$ can be split as $f=u+\overline{e_{0}} v$ where $u$ and $v$ are $\mathbb{R}_{0, m}$-valued; sometimes $u$ and $v$ are referred to as the real and the imaginary parts of the function $f$. By $\partial$ we now denote the Dirac operator in $\mathbb{R}^{m+1}$, i.e. $\partial=\sum_{\alpha=0}^{m} e_{\alpha} \partial_{X_{\alpha}}=e_{0} \partial_{X_{0}}+\underline{\partial}$. If $f=u+\overline{e_{0}} v$ is monogenic in a region $\Omega$ of $\mathbb{R}^{m+1}$, i.e. $\partial f=0$ in $\Omega$, then $u$ and $v$ are harmonic functions in $\Omega$ satisfying the system

$$
\left\{\begin{array}{l}
\partial_{X_{0}} v+\underline{\partial} u=0 \\
\underline{\partial} v+\partial_{X_{0}} u=0
\end{array}\right.
$$

which can be seen as a generalization of the classical Cauchy-Riemann system in the complex plane. The pair $(u, v)$ is then called a pair of conjugate harmonics in $\Omega$. The question under which geometric condition on $\Omega$ the monogenic function $f$ is determined, up to an $\mathbb{R}_{0, m}$-valued monogenic function, by its harmonic first component $u$, was solved in a constructive way in [5] as follows. 
Definition 1. Let $\Omega \subset \mathbb{R}^{m+1}$ be open and let $\widetilde{\Omega}$ be its projection on $\mathbb{R}^{m}$ along the $e_{0}$-direction. Then $\Omega$ is called $e_{0}$-normal if there exists an $X_{0}^{*} \in \mathbb{R}$ such that for all $\underline{X} \in \widetilde{\Omega}$, the intersection $\Omega \cap\left\{t e_{0}+\underline{X} \mid t \in \mathbb{R}\right\} \neq \emptyset$ is connected and contains the point $X_{0}^{*} e_{0}+\underline{X}$.

Theorem 1. Let $\Omega \subset \mathbb{R}^{m+1}$ be an $e_{0}$-normal region and let $u$ be an $\mathbb{R}_{0, m}$-valued harmonic function in $\Omega$. Then there exists an $\mathbb{R}_{0, m}$-valued harmonic potential $H$ in $\Omega$ such that

(i) $v=-\underline{\partial} H$ is an $\mathbb{R}_{0, m}$-valued conjugate harmonic in $\Omega$ to $u=\partial_{X_{0}} H$;

(ii) $f=u+\overline{e_{0}} v=\partial \overline{e_{0}} H$ is monogenic in $\Omega$.

Remark 1. If, in particular, $u$ is a real-valued harmonic function in $\Omega$, then the harmonic potential $H$ is also real-valued, the conjugate harmonic $v=\sum_{j=1}^{m} e_{j} v_{j}$ is Clifford vector-valued and the vector field

$$
F^{*}=\left[u,-v_{1},-v_{2}, \ldots,-v_{m}\right]
$$

is an $(m+1)$-tuple of conjugate harmonics in the sense of Stein-Weiss, for which $F^{*}=\operatorname{grad} H$.

Also the question of the monogenic primitive has been settled in the Clifford analysis framework. Introducing the Cauchy-Riemann operator $D=\overline{e_{0}} \partial=\partial_{X_{0}}+\overline{e_{0}} \underline{\partial}$ and its Clifford conjugate $\bar{D}=-\partial e_{0}=\partial_{X_{0}}-\overline{e_{0}} \underline{\partial}$, which factorize the Laplace operator: $D \bar{D}=\bar{D} D=\Delta_{m+1}$, we have the following result.

Theorem 2. Let $\Omega \subset \mathbb{R}^{m+1}$ be an $e_{0}$-normal region and let $f$ be monogenic in $\Omega$. Then the function $F$ given by

$$
F\left(X_{0}, \underline{X}\right)=\frac{1}{2} \int_{X_{0}^{*}}^{X_{0}} f(t, \underline{X}) d t+\frac{1}{2} \overline{e_{0}} \underline{\partial g}(\underline{X})
$$

where the function $g$ satisfies the equation $\Delta_{m} g(\underline{X})=f\left(X_{0}^{*}, \underline{X}\right)$, is a monogenic primitive of $f$ in $\Omega$, satisfying there $D F=0$ and $\bar{D} F=f$.

Note that, as is the case in the complex plane, a monogenic primitive $F=U+\overline{e_{0}} V$ of the monogenic function $f=u+\overline{e_{0}} v$ in $\Omega$ provides automatically two conjugate harmonic primitives $2 U$ and $2 \overline{e_{0}} V$ in the same region, satisfying $\bar{D}(2 U)=\bar{D}\left(2 \overline{e_{0}} V\right)=f$ and also $u=\partial_{X_{0}}(2 U)=\underline{\partial}(-2 V), v=$ $\partial_{X_{0}}(2 V)=\underline{\partial}(-2 U)$.

Even when the region $\Omega$ is not $e_{0}$-normal, it is still possible to construct locally a harmonic primitive of a given monogenic function in $\Omega$. Indeed, we have the following result involving the conjugate Teodorescu transform, the Teodorescu transform itself being a right inverse of the Cauchy-Riemann operator $D$.

Lemma 2. Let $f$ be monogenic in the region $\Omega \subset \mathbb{R}^{m+1}$. For each bounded open $\Omega_{0} \subset \Omega$ such that $\overline{\Omega_{0}}$ is compact and contained in $\Omega$, the function $\widetilde{F}$ given by

$$
\widetilde{F}\left(X_{0}, \underline{X}\right)=\int_{\Omega_{0}} E\left(Y_{0}-X_{0}, \underline{Y}-\underline{X}\right) f\left(Y_{0}, \underline{Y}\right) d V_{Y}
$$

with

$$
E\left(X_{0}, \underline{X}\right)=\frac{1}{a_{m+1}} \frac{X_{0}+\overline{e_{0}} \underline{X}}{\left|X_{0}+\overline{e_{0}} \underline{X}\right|^{m+1}}
$$

and $a_{m+1}$ the area of the unit sphere in $\mathbb{R}^{m+1}$, is a harmonic primitive of $f$ in $\Omega_{0}$, satisfying there $\Delta_{m+1} \widetilde{F}=0$ and $\bar{D} \widetilde{F}=f$. 
In the books $[26,16]$ and the series of papers $[27,18,1,2,11,19,8]$ so-called Hermitean Clifford analysis recently emerged as a refinement of Euclidean Clifford analysis, where the considered functions now take their values in the complex Clifford algebra $\mathbb{C}_{m}$ or in complex spinor space. Hermitean Clifford analysis is based on the introduction of an additional datum, a so-called complex structure $J$, inducing an associated Dirac operator $\underline{\partial}_{J}$; it then focusses on the simultaneous null solutions of both operators $\underline{\partial}$ and $\underline{\partial}_{J}$, called Hermitean monogenic functions. The corresponding function theory is still in full development, see also $[7,12,28,4,3,20,13$, 10]. It is worth mentioning that the traditional holomorphic functions of several complex variables are a special case of Hermitean monogenic functions.

The aim of this paper is (i) to introduce the notion of a conjugate pair of harmonics summing up to a Hermitean monogenic function, (ii) to construct in this Hermitean Clifford analysis context the harmonic conjugate to a given harmonic function and (iii) to determine, under certain geometric constraints on the domain considered, the potential or primitive of a Hermitean monogenic function.

\section{Preliminaries on Clifford analysis}

For a detailed description of the structure of Clifford algebras we refer to e.g. [25]. Here we only recall the necessary basic notions. The real Clifford algebra $\mathbb{R}_{0, m}$ is constructed over the vector space $\mathbb{R}^{0, m}$ endowed with a non-degenerate quadratic form of signature $(0, m)$ and generated by the orthonormal basis $\left(e_{1}, \ldots, e_{m}\right)$. The non-commutative Clifford or geometric multiplication in $\mathbb{R}_{0, m}$ is governed by the rules

$$
e_{\alpha} e_{\beta}+e_{\beta} e_{\alpha}=-2 \delta_{\alpha \beta} \quad, \quad \alpha, \beta=1, \ldots, m
$$

As a basis for $\mathbb{R}_{0, m}$ one takes for any set $A=\left\{j_{1}, \ldots, j_{h}\right\} \subset\{1, \ldots, m\}$ the element $e_{A}=e_{j_{1}} \ldots e_{j_{h}}$, with $1 \leq j_{1}<j_{2}<\cdots<j_{h} \leq m$, together with $e_{\emptyset}=1$, the identity element. Any Clifford number $a$ in $\mathbb{R}_{0, m}$ may thus be written as $a=\sum_{A} e_{A} a_{A}, a_{A} \in \mathbb{R}$, or still as $a=\sum_{k=0}^{m}[a]_{k}$, where $[a]_{k}=\sum_{|A|=k} e_{A} a_{A}$ is the so-called $k$-vector part of $a$. Euclidean space $\mathbb{R}^{0, m}$ is embedded in $\mathbb{R}_{0, m}$ by identifying $\left(X_{1}, \ldots, X_{m}\right)$ with the Clifford vector $\underline{X}=\sum_{\alpha=1}^{m} e_{\alpha} X_{\alpha}$, for which it holds that $\underline{X}^{2}=-|\underline{X}|^{2}$. The vector-valued first order differential operator $\underline{\partial}=\sum_{\alpha=1}^{m} e_{\alpha} \partial_{X_{\alpha}}$, called Dirac operator, is the Fourier or Fischer dual of the Clifford variable $\underline{X}$. It is this operator which underlies the notion of monogenicity of a function, a notion which is the higher dimensional counterpart of holomorphy in the complex plane. More explicitly, a function $f$ defined and continuously differentiable in an open region $\Omega$ of $\mathbb{R}^{m}$ and taking values in (a subspace of) the Clifford algebra $\mathbb{R}_{0, m}$, is called (left) monogenic in $\Omega$ if $\underline{\partial}[f]=0$ in $\Omega$. As the Dirac operator factorizes the Laplace operator: $\Delta_{m}=-\underline{\partial}^{2}$, monogenicity can be regarded as a refinement of harmonicity. The Dirac operator being rotationally invariant, or more precisely: invariant under the orthogonal group $\mathrm{O}(m ; \mathbb{R})$, which is doubly covered by the $\operatorname{Pin}(m)$ group of the Clifford algebra, this framework is usually referred to as Euclidean Clifford analysis.

When allowing for complex constants, the generators $\left(e_{1}, \ldots, e_{m}\right)$, still satisfying $(2.1)$, produce the complex Clifford algebra $\mathbb{C}_{m}=\mathbb{R}_{0, m} \oplus i \mathbb{R}_{0, m}$. Any complex Clifford number $\lambda \in \mathbb{C}_{m}$ may thus be written as $\lambda=a+i b, a, b \in \mathbb{R}_{0, m}$, leading to the definition of the Hermitean conjugation $\lambda^{\dagger}=(a+i b)^{\dagger}=\bar{a}-i \bar{b}$, where the bar notation stands for the Clifford conjugation in $\mathbb{R}_{0, m}$, i.e. the main anti-involution for which $\bar{e}_{\alpha}=-e_{\alpha}, \alpha=1, \ldots, m$. This Hermitean conjugation leads to a Hermitean inner product on $\mathbb{C}_{m}$ given by $(\lambda, \mu)=\left[\lambda^{\dagger} \mu\right]_{0}$ and its associated norm $|\lambda|=\sqrt{\left[\lambda^{\dagger} \lambda\right]_{0}}=\left(\sum_{A}\left|\lambda_{A}\right|^{2}\right)^{1 / 2}$. This is the framework for Hermitean Clifford analysis, which emerges from Euclidean Clifford analysis by introducing an additional datum, a so-called complex structure, i.e. an $\mathrm{SO}(m ; \mathbb{R})$-element $J$ with $J^{2}=\mathbf{- 1}$ (see $[1,2]$ ), forcing the dimension to be 
even: from now on we put $m=2 n$. Usually $J$ is chosen to act upon the generators of $\mathbb{C}_{2 n}$ as $J\left[e_{j}\right]=-e_{n+j}$ and $J\left[e_{n+j}\right]=e_{j}, j=1, \ldots, n$. By means of the projection operators $\pm \frac{1}{2}(\mathbf{1} \pm i J)$ associated to $J$, first the Witt basis elements $\left(\mathfrak{f}_{j}, \mathfrak{f}_{j}^{\dagger}\right)_{j=1}^{n}$ for $\mathbb{C}_{2 n}$ are obtained:

$$
\begin{aligned}
\mathfrak{f}_{j} & =\frac{1}{2}(\mathbf{1}+i J)\left[e_{j}\right]=\frac{1}{2}\left(e_{j}-i e_{n+j}\right), & & j=1, \ldots, n \\
\mathfrak{f}_{j}^{\dagger}=-\frac{1}{2}(\mathbf{1}-i J)\left[e_{j}\right] & =-\frac{1}{2}\left(e_{j}+i e_{n+j}\right), & & j=1, \ldots, n
\end{aligned}
$$

The Witt basis elements satisfy the respective Grassmann and duality identities

$$
\mathfrak{f}_{j} \mathfrak{f}_{k}+\mathfrak{f}_{k} \mathfrak{f}_{j}=\mathfrak{f}_{j}^{\dagger} \mathfrak{f}_{k}^{\dagger}+\mathfrak{f}_{k}^{\dagger} \mathfrak{f}_{j}^{\dagger}=0, \quad \mathfrak{f}_{j} \mathfrak{f}_{k}^{\dagger}+\mathfrak{f}_{k}^{\dagger} \mathfrak{f}_{j}=\delta_{j k}, \quad j, k=1, \ldots, n
$$

whence they are isotropic: $\left(\mathfrak{f}_{j}\right)^{2}=0,\left(\mathfrak{f}_{j}^{\dagger}\right)^{2}=0, j=0, \ldots, n$. Next, a vector in $\mathbb{R}^{0,2 n}$ is now denoted by $\left(x_{1}, \ldots, x_{n}, y_{1}, \ldots, y_{n}\right)$ and identified with the Clifford vector $\underline{X}=\sum_{j=1}^{n}\left(e_{j} x_{j}+e_{n+j} y_{j}\right)$, producing, by projection, the Hermitean Clifford variables $\underline{z}$ and $\underline{z}^{\dagger}$ :

$$
\underline{z}=\frac{1}{2}(\mathbf{1}+i J)[\underline{X}]=\sum_{j=1}^{n} \mathfrak{f}_{j} z_{j}, \quad \underline{z}^{\dagger}=-\frac{1}{2}(\mathbf{1}-i J)[\underline{X}]=\sum_{j=1}^{n} \mathfrak{f}_{j}^{\dagger} z_{j}^{c}
$$

where complex variables $z_{j}=x_{j}+i y_{j}$ have been introduced, with complex conjugates $z_{j}^{c}=x_{j}-i y_{j}$, $j=1, \ldots, n$. Finally, the Euclidean Dirac operator $\underline{\partial}$ gives rise, in the same way, to the Hermitean Dirac operators $\partial_{\underline{z}}$ and $\partial_{\underline{z}}^{\dagger}$ :

$$
\partial_{\underline{z}}^{\dagger}=\frac{1}{4}(\mathbf{1}+i J)[\underline{\partial}]=\sum_{j=1}^{n} \mathfrak{f}_{j} \partial_{z_{j}^{c}}, \quad \partial_{\underline{z}}=-\frac{1}{4}(\mathbf{1}-i J)[\underline{\partial}]=\sum_{j=1}^{n} \mathfrak{f}_{j}^{\dagger} \partial_{z_{j}}
$$

involving the Cauchy-Riemann operators $\partial_{\bar{z}_{j}}=\frac{1}{2}\left(\partial_{x_{j}}+i \partial_{y_{j}}\right)$ and their complex conjugates $\partial_{z_{j}}=$ $\frac{1}{2}\left(\partial_{x_{j}}-i \partial_{y_{j}}\right)$ in the $z_{j}$-planes, $j=1, \ldots, n$. Observe that Hermitean vector variables and Dirac operators are isotropic, i.e. $\underline{z}^{2}=\left(\underline{z}^{\dagger}\right)^{2}=0$ and $\left(\partial_{\underline{z}}\right)^{2}=\left(\partial_{\underline{z}}^{\dagger}\right)^{2}=0$, whence the Laplace operator allows for the decomposition and factorization

$$
\Delta_{2 n}=4\left(\partial_{\underline{z}} \partial_{\underline{z}}^{\dagger}+\partial_{\underline{z}}^{\dagger} \partial_{\underline{z}}\right)=4\left(\partial_{\underline{z}}+\partial_{\underline{z}}^{\dagger}\right)^{2}=-4\left(\partial_{\underline{z}}^{\dagger}-\partial_{\underline{z}}\right)^{2}
$$

while dually

$$
-\left(\underline{z}-\underline{z}^{\dagger}\right)^{2}=\left(\underline{z}+\underline{z}^{\dagger}\right)^{2}=\underline{z} \underline{z}^{\dagger}+\underline{z}^{\dagger} \underline{z}=|\underline{z}|^{2}=\left|\underline{z}^{\dagger}\right|^{2}=|\underline{X}|^{2}
$$

A continuously differentiable function $g$ in a region, i.e. an open connected set $\Omega$ of $\mathbb{R}^{2 n}$ with values in (a subspace of) the complex Clifford algebra $\mathbb{C}_{2 n}$ then is called (left) Hermitean monogenic (or h-monogenic) in $\Omega$ if and only if it satisfies in $\Omega$ the system $\partial_{\underline{z}} g=0=\partial_{\underline{z}}^{\dagger} g$, or, equivalently, the system $\underline{\partial} g=\underline{\partial}_{J} g$, with $\underline{\partial}_{J}=J[\underline{\partial}]$. A major difference between Hermitean and Euclidean Clifford analysis concerns the underlying group invariance. Where $\underline{\partial}$ is invariant under the action of $\mathrm{SO}(m)$, the system invariance of $\left(\partial_{\underline{z}}, \partial_{\underline{z}}^{\dagger}\right)$ breaks down to the unitary group $\mathrm{U}(n)$, see e.g. [1, 2]. For this reason $\mathrm{U}(n)$ plays a fundamental role in the development of the Hermitean function theory.

\section{Conjugate pairs in the Hermitean framework}

We consider functions $g\left(z_{1}, \ldots, z_{n}, \bar{z}_{1}, \ldots, \bar{z}_{n}\right)$, defined in a region $\Omega$ of $\mathbb{R}^{2 n} \simeq \mathbb{C}^{n}$ and taking values in spinor space $\mathbb{S}_{n}$ realized as $\mathbb{S}_{n}=\bigwedge^{\dagger}\left(\mathfrak{f}_{1}^{\dagger}, \ldots, \mathfrak{f}_{n}^{\dagger}\right) I, I$ being the self-adjoint idempotent given 
by $I=\mathfrak{f}_{1} \mathfrak{f}_{1}^{\dagger} \ldots \mathfrak{f}_{n} \mathfrak{f}_{n}^{\dagger}$. In retrospect the notion of conjugate harmonic pair in Euclidean Clifford analysis was made possible by the splitting of the value space $\mathbb{R}_{0, m+1}$ into two equivalent parts: $\mathbb{R}_{0, m+1}=\mathbb{R}_{0, m} \oplus \overline{e_{0}} \mathbb{R}_{0, m}$, mimicing the splitting of the complex plane as $\mathbb{C}=\mathbb{R} \oplus i \mathbb{R}$. So it is clear that also in the Hermitean Clifford setting we will need a splitting of the value space into two equivalent parts. To that end we single out the Witt basis vectors $\mathfrak{f}_{n}$ and $\mathfrak{f}_{n}^{\dagger}$, and we decompose spinor space as

$$
\mathbb{S}_{n}=\bigwedge^{\dagger}\left(\mathfrak{f}_{1}^{\dagger}, \ldots, \mathfrak{f}_{n-1}^{\dagger}\right) I \oplus \mathfrak{f}_{n}^{\dagger} \bigwedge^{\dagger}\left(\mathfrak{f}_{1}^{\dagger}, \ldots, \mathfrak{f}_{n-1}^{\dagger}\right) I
$$

Accordingly the functions under consideration are rewritten as $g=g^{0}+\mathfrak{f}_{n}^{\dagger} g^{1}$, where both $g^{0}$ and $g^{1}$ take their values in $\Lambda^{\dagger}\left(\mathfrak{f}_{1}^{\dagger}, \ldots, \mathfrak{f}_{n-1}^{\dagger}\right) I$. In the same order of ideas the Hermitean variables and the Hermitean Dirac operators are decomposed as

$$
\begin{aligned}
\underline{z}=\underline{\widetilde{z}}+\mathfrak{f}_{n} z_{n}, & \underline{\widetilde{z}}=\sum_{j=1}^{n-1} \mathfrak{f}_{j} z_{j} \\
\underline{z}^{\dagger}=\underline{\widetilde{z}}^{\dagger}+\mathfrak{f}_{n}^{\dagger} \bar{z}_{n}, & \underline{\widetilde{z}}^{\dagger}=\sum_{j=1}^{n-1} \mathfrak{f}_{j}^{\dagger} \bar{z}_{j} \\
\partial_{\underline{z}}=\widetilde{\partial_{\underline{z}}}+\mathfrak{f}_{n}^{\dagger} \partial_{z_{n}}, & \widetilde{\partial_{\underline{z}}}=\sum_{j=1}^{n-1} \mathfrak{f}_{j}^{\dagger} \partial_{z_{j}} \\
\partial_{\underline{z}}^{\dagger}=\widetilde{\partial_{\underline{z}}^{\dagger}}+\mathfrak{f}_{n} \partial_{\bar{z}_{n}}, & \widetilde{\partial_{\underline{z}}^{\dagger}}=\sum_{j=1}^{n-1} \mathfrak{f}_{j} \partial_{\bar{z}_{j}}
\end{aligned}
$$

Definition 2. If $g: \Omega \longrightarrow \mathbb{S}_{n}$ is Hermitean monogenic in $\Omega$, and we write $g$ as $g^{0}+\mathfrak{f}_{n}^{\dagger} g^{1}$, then the ordered pair $\left(g^{0}, g^{1}\right)$ is called a Hermitean conjugate pair in $\Omega$.

The properties of the conjugate pair $\left(g^{0}, g^{1}\right)$ will follow from the Hermitean monogenicity of the function $g$. From $\partial_{\underline{z}} g=0$ in $\Omega$ we obtain $\widetilde{\partial}_{\underline{z}} g^{0}+\mathfrak{f}_{n}^{\dagger} \partial_{z_{n}} g^{0}+\widetilde{\partial}_{\underline{z}} f_{n}^{\dagger} g^{1}=0$, or

$$
\begin{gathered}
\widetilde{\partial}_{\underline{z}} g^{0}=0 \\
\partial_{z_{n}} g^{0}-\widetilde{\partial}_{\underline{z}} g^{1}=0
\end{gathered}
$$

seen the different value spaces of both expressions. In a similar way it follows from $\partial_{\underline{z}}^{\dagger} g=0$ in $\Omega$ that

$$
\begin{gathered}
\widetilde{\partial_{\underline{z}}^{\dagger}} g^{0}+\partial_{\bar{z}_{n}} g^{1}=0 \\
\widetilde{\partial_{\underline{z}}^{\dagger}} g^{1}=0
\end{gathered}
$$

We can make the following observations.

(a) As $g$ is Hermitean monogenic and hence monogenic in $\Omega$, it is harmonic, and so are the components $g^{0}$ and $g^{1}$, since the Laplace operator is a scalar operator.

(b) The function $g^{0}$ always belongs to Ker $\widetilde{\partial}_{\underline{z}}$, but it cannot be Hermitean monogenic w.r.t. $\widetilde{\partial}_{\underline{z}}$ and $\widetilde{\partial_{\underline{z}}^{\dagger}}$ unless $g^{1}$ does not depend on the variable $\bar{z}_{n}$.

(c) The function $g^{1}$ always belongs to Ker $\widetilde{\partial_{\underline{z}}^{\dagger}}$, but it cannot be Hermitean monogenic w.r.t. $\widetilde{\partial_{z}}$ and $\widetilde{\partial_{\underline{z}}^{\dagger}}$ unless $g^{0}$ does not depend on the variable $z_{n}$. 
(d) Combining the relations (3.2) and (3.3) we obtain

$$
\widetilde{\partial_{\underline{z}}} \widetilde{\partial_{\underline{z}}^{\dagger}} g^{0}+\partial_{z_{n}} \partial_{\bar{z}_{n}} g^{0}=\left[\widetilde{\partial_{\underline{z}}} \widetilde{\partial_{\underline{z}}^{\dagger}}+\left(\mathfrak{f}_{n} \partial_{\bar{z}_{n}}\right)\left(\mathfrak{f}_{n}^{\dagger} \partial_{z_{n}}\right)\right] g^{0}=0
$$

and also

$$
\widetilde{\partial_{\underline{z}}^{\dagger}} \widetilde{\partial}_{\underline{z}} g^{1}+\partial_{z_{n}} \partial_{\bar{z}_{n}} g^{1}=\left[\widetilde{\partial_{\underline{z}}^{\dagger}} \widetilde{\partial}_{\underline{z}}+\left(\mathfrak{f}_{n} \partial_{\bar{z}_{n}}\right)\left(\mathfrak{f}_{n}^{\dagger} \partial_{z_{n}}\right)\right] g^{1}=0
$$

(e) Combining (3.1) and (3.5) we obtain

$$
\begin{aligned}
& \frac{1}{4} \Delta g^{0}=\left(\partial_{\underline{z}}^{\dagger} \partial_{\underline{z}}+\partial_{\underline{z}} \partial_{\underline{z}}^{\dagger}\right) g^{0}=\left(\widetilde{\partial_{\underline{z}}} \widetilde{\partial_{\underline{z}}^{\dagger}}+\mathfrak{f}_{n}^{\dagger} \partial_{z_{n}} \widetilde{\partial_{\underline{z}}^{\dagger}}+\widetilde{\partial_{\underline{z}}^{\dagger}} \widetilde{\partial_{z}}+\widetilde{\partial_{\underline{z}}^{\dagger}} f_{n}^{\dagger} \partial_{z_{n}}+\mathfrak{f}_{n} \mathfrak{f}_{n}^{\dagger} \partial_{\bar{z}_{n}} \partial_{z_{n}}\right) g^{0} \\
& =\left(\widetilde{\partial_{\underline{z}}} \widetilde{\partial_{\underline{z}}^{\dagger}}+\mathfrak{f}_{n} f_{n}^{\dagger} \partial_{\bar{z}_{n}} \partial_{z_{n}}\right) g^{0}=0
\end{aligned}
$$

confirming the harmonicity of $g^{0}$. Similarly, (3.4) and (3.6) imply the harmonicity of $g^{1}$.

(f) The system (3.2)-(3.3) can be seen as the CR-system in the Hermitean Clifford analysis context, and so the following problem arises quite naturally.

\section{Problem}

Given in the open region $\Omega \subset \mathbb{C}^{n}$ the harmonic function $g^{0}$ with values in $\bigwedge^{\dagger}\left(\mathfrak{f}_{1}^{\dagger}, \ldots, \mathfrak{f}_{n-1}^{\dagger}\right) I$, and which moreover belongs to Ker $\widetilde{\widetilde{z}}_{\underline{z}}$, find the harmonic function $g^{1}$ also with values in $\Lambda^{\dagger}\left(\mathfrak{f}_{1}^{\dagger}, \ldots, \mathfrak{f}_{n-1}^{\dagger}\right) I$ and moreover belonging to Ker $\partial_{z}^{\dagger}$, such that $\left(g^{0}, g^{1}\right)$ is a Hermitean conjugate pair, i.e. such that the function $g=g^{0}+\mathrm{f}_{n}^{\dagger} g^{1}$ is Hermitean monogenic in $\Omega$.

This problem clearly has no unique solution, since a Hermitean monogenic function with values in $\mathfrak{f}_{n}^{\dagger} \bigwedge^{\dagger}\left(\mathfrak{f}_{1}^{\dagger}, \ldots, \mathfrak{f}_{n-1}^{\dagger}\right) I$ can always be added to the second term. We will now solve this problem in a constructive way at least locally.

Take the function $g^{0} \in \operatorname{Ker} \widetilde{\partial}_{\underline{z}} \cap \operatorname{Ker} \Delta$, i.e. $g^{0}$ satisfies conditions (3.1), (3.7), and also (3.5). Moreover, consider a bounded open region $\Omega_{0} \subset \mathbb{C}^{n}$ such that its closure $\bar{\Omega}_{0}$ is contained in $\Omega$ and, in addition, $\Omega_{0}=\Omega_{n} \times \widetilde{\Omega}$ for some open regions $\Omega_{n}$ in the complex $z_{n}$-plane and $\widetilde{\Omega}$ in $\mathbb{C}^{n-1}$. For the moment, $\widetilde{\Omega}$ is not further specified, but it is clear that for each $\underline{\widetilde{z}}_{*} \in \widetilde{\Omega}$, the corresponding variables $z_{n}$ for which $\left(\widetilde{z}_{*}, z_{n}\right) \in \Omega_{0}$, belong to the same region $\Omega_{n}$. Then we want to solve equation (3.3) for $g^{1}$ on $\Omega_{0}$. In fact this is a problem in the complex $z_{n}$-plane, for which we can use the following lemma.

Lemma 3 (see $[24,1.1 .3])$. Let $G \subset \mathbb{C}$ be a bounded open region, let $f \in C_{\infty}(G)$ be a bounded complex-valued function and let $T_{G} f$ be its Pompeiu transform, given by

$$
T_{G} f(z)=-\frac{1}{2 \pi i} \int_{G} \frac{f(w)}{w-z} d \bar{w} \wedge d w, z \in G
$$

Then $F:=T_{G} f$ belongs to $C_{\infty}(G)$ and solves the equation $\partial_{\bar{z}} F=f$.

Using Lemma 3 , it is easy to see that the equation (3.3) has a solution $G^{1}\left(z_{n}, \bar{z}_{n}, \underline{\widetilde{z}}, \underline{z}^{\dagger}\right) \in C_{\infty}\left(\Omega_{0}\right)$ and all solutions of (3.3) are given by

$$
g^{1}=G^{1}+h^{1}\left(z_{n}, \underline{\widetilde{z}}, \underline{z}^{\dagger}\right)
$$


where the following conditions are imposed on the function $h^{1}$, since the function $g^{1}$, defined by (3.8), should satisfy (3.2) and (3.4):

$$
\begin{gathered}
\partial_{z_{n}} g^{0}=\widetilde{\partial_{\underline{z}}} G^{1}+\widetilde{\partial_{\underline{z}}} h^{1} \\
\widetilde{\partial_{\underline{z}}^{\dagger}} G^{1}+\widetilde{\partial_{\underline{z}}^{\dagger}} h^{1}=0
\end{gathered}
$$

Note that in condition (3.9) the variable $\bar{z}_{n}$ does not occur since

$$
\partial_{\bar{z}_{n}} \partial_{z_{n}} g^{0}-\partial_{\bar{z}_{n}} \widetilde{\partial}_{\underline{z}} G^{1}=\partial_{\bar{z}_{n}} \partial_{z_{n}} g^{0}+\widetilde{\partial_{\underline{z}}} \widetilde{\partial_{\underline{z}}^{\dagger}} g^{0}=0
$$

due to (3.5). In order for equation (3.9) to have a $C_{\infty}\left(\Omega_{0}\right)$ solution $h^{1}\left(z_{n}, \underline{\widetilde{z}}, \underline{\widetilde{z}}^{\dagger}\right)$, a certain geometric condition on $\widetilde{\Omega}$ will have to be imposed. This issue will be explicitly addressed in Section 4. It turns out that it is sufficient to assume that $\widetilde{\Omega}$ is strictly pseudoconvex (see Lemma 3 ). The particular form of $\Omega_{0}$ ensures that for each $z_{n}^{*} \in \Omega_{n}$ fixed, the corresponding variables $\underline{\widetilde{z}}$ for which $\left(\underline{z}, z_{n}\right) \in \Omega_{0}$ belong to the same region $\widetilde{\Omega}$. Also note that, seen the values taken by the function $G^{1}$, there holds $\widetilde{\partial_{\underline{z}}^{\dagger}} G^{1}=0$, which reduces equation (3.10) to $\widetilde{\partial_{\underline{z}}^{\dagger}} h^{1}=0$.

We thus have proven the following theorem.

Theorem 3. Let $\Omega$ be a region in $\mathbb{C}^{n}$. Let the function

$$
g^{0}: \Omega \rightarrow \bigwedge^{\dagger}\left(\mathfrak{f}_{1}^{\dagger}, \ldots, \mathfrak{f}_{n-1}^{\dagger}\right) I
$$

be harmonic and belonging to Ker $\widetilde{\partial_{\underline{z}}}$, i.e. $\widetilde{\partial_{\underline{z}}} g^{0}=0$ and $\widetilde{\partial_{\underline{z}}} \widetilde{\partial_{\underline{z}}^{\dagger}} g^{0}+\partial_{z_{n}} \partial_{\bar{z}_{n}} g^{0}=0$. Then there exists locally in $\Omega$ a harmonic function $g^{1}$ with values in $\Lambda^{\dagger}\left(\mathrm{f}_{1}^{\dagger}, \ldots, \mathrm{f}_{n-1}^{\dagger}\right) I$ and moreover belonging to $\operatorname{Ker} \widetilde{\partial_{\underline{z}}^{\dagger}}$, such that $\left(g^{0}, g^{1}\right)$ is a Hermitean conjugate pair.

More explicitly, let $\Omega_{0}=\Omega_{n} \times \widetilde{\Omega}$ for some bounded region $\Omega_{n}$ in the complex $z_{n}$-plane and some bounded strictly pseudoconvex open set $\widetilde{\Omega}$ in $\mathbb{C}^{n-1}$ such that $\bar{\Omega}_{0} \subset \Omega$. Then there are solutions $G^{1}$ and $h^{1}$ in $C_{\infty}\left(\Omega_{0}\right)$ of the following system of equations

$$
\partial_{\bar{z}_{n}} G^{1}=-\widetilde{\partial_{\underline{z}}^{\dagger}} g^{0}, \quad \partial_{\bar{z}_{n}} h^{1}=0, \quad \widetilde{\partial_{\underline{z}}} h^{1}=\partial_{z_{n}} g^{0}-\widetilde{\partial_{\underline{z}}} G^{1}, \quad \widetilde{\partial_{\underline{z}}^{\dagger}} h^{1}=0 .
$$

Moreover, the function $g^{1}:=G^{1}+h^{1}$ enjoys the following properties in $\Omega_{0}$ :

(i) $\widetilde{\partial_{\underline{z}}^{\dagger}} g^{1}=0, \widetilde{\partial_{\underline{z}}} g^{1}=\partial_{z_{n}} g^{0}, \partial_{\bar{z}_{n}} g^{1}=-\widetilde{\partial_{\underline{z}}^{\dagger}} g^{0}$;

(ii) $g^{1}$ is harmonic;

(iii) $g^{0}+\mathfrak{f}_{n}^{\dagger} g^{1}$ is Hermitean monogenic.

Let us illustrate this construction of a Hermitean conjugate pair by the following example. Given is the function $g^{0}=\bar{z}_{2} z_{3}^{2} \mathfrak{f}_{1}^{\dagger} I$ in $\mathbb{R}^{6} \simeq \mathbb{C}^{3}$. We verify that $g^{0}$ takes its values in $\bigwedge\left(\mathfrak{f}_{1}^{\dagger}, \mathfrak{f}_{2}^{\dagger}\right) I$ and satisfies $\widetilde{\partial_{\underline{z}}} g^{0}=0, \widetilde{\partial_{\underline{z}}} \widetilde{\partial_{\underline{z}}^{\dagger}} g^{0}+\partial_{z_{3}} \partial_{\bar{z}_{3}} g^{0}=0$. As also $\widetilde{\partial_{\underline{z}}^{\dagger}} g^{0}=0$, we put $g^{1}=h\left(z_{3}, \widetilde{\widetilde{z}}, \widetilde{z}^{\dagger}\right)$ taking values in $\bigwedge\left(\mathfrak{f}_{1}^{\dagger}, \mathfrak{f}_{2}^{\dagger}\right) I$. This function $h$ is subject to the conditions $\widetilde{\partial_{\underline{z}}^{\dagger}} h=0$ and $\widetilde{\partial}_{\underline{z}} h=2 \bar{z}_{2} z_{3} \mathfrak{f}_{1}^{\dagger} I$. If we put

$$
h\left(z_{3}, \underline{\widetilde{z}}, \underline{z}^{\dagger}\right)=h_{0} I+h_{1} \mathfrak{f}_{1}^{\dagger} I+h_{2} \mathfrak{f}_{2}^{\dagger} I+h_{12} \mathfrak{f}_{1}^{\dagger} \mathfrak{f}_{2}^{\dagger} I
$$

we are lead to the systems

$$
\left\{\begin{array} { l } 
{ \partial _ { z _ { 1 } } h _ { 0 } = 2 \overline { z } _ { 2 } z _ { 3 } } \\
{ \partial _ { z _ { 2 } } h _ { 0 } = 0 } \\
{ \partial _ { z _ { 1 } } h _ { 2 } - \partial _ { z _ { 2 } } h _ { 1 } = 0 }
\end{array} \quad \text { and } \quad \left\{\begin{array}{l}
\partial_{\bar{z}_{1}} h_{1}+\partial_{\bar{z}_{2}} h_{2}=0 \\
\partial_{\bar{z}_{1}} h_{12}=0 \\
\partial_{\bar{z}_{2}} h_{12}=0
\end{array}\right.\right.
$$


from which it follows that $h_{0}=\left(2 z_{1} \bar{z}_{2} z_{3}+k\left(\bar{z}_{1}, \bar{z}_{2}, z_{3}\right)\right) I$. The function $g^{1}$ takes the form

$$
g^{1}=2 z_{1} \bar{z}_{2} z_{3} I+k\left(\bar{z}_{1}, \bar{z}_{2}, z_{3}\right) I+\left(h_{1} \mathrm{f}_{1}^{\dagger} I+h_{2} \mathrm{f}_{2}^{\dagger} I\right)+h_{12} \mathrm{f}_{1}^{\dagger} \mathrm{f}_{2}^{\dagger} I
$$

with $h_{1}$ and $h_{2}$ satisfying

$$
\left\{\begin{array}{l}
\partial_{z_{1}} h_{2}-\partial_{z_{2}} h_{1}=0 \\
\partial_{\bar{z}_{1}} h_{1}+\partial_{\bar{z}_{2}} h_{2}=0
\end{array}\right.
$$

and $h_{12}$ satisfying $\partial_{\bar{z}_{1}} h_{12}=\partial_{\bar{z}_{2}} h_{12}=0$. It is then indeed seen that the function

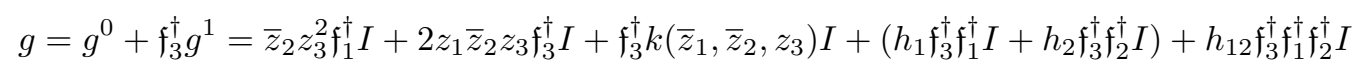

is Hermitean monogenic in $\mathbb{C}^{3}$.

A similar theorem may be formulated starting with the function $g^{1} \in \operatorname{Ker} \widetilde{\partial_{\underline{z}}^{\dagger}} \cap \operatorname{Ker} \Delta$.

Theorem 4. Let $\Omega$ be an open region in $\mathbb{C}^{n}$ of the form $\Omega=\Omega_{n} \times \widetilde{\Omega}$, where $\Omega_{n}$ is an open simply connected region in the complex $z_{n}$-plane, and $\widetilde{\Omega}$ is an open pseudoconvex domain in $\mathbb{C}^{n-1}$. Let the function $g^{1}: \Omega \rightarrow \Lambda^{\dagger}\left(\mathfrak{f}_{1}^{\dagger}, \ldots, \mathfrak{f}_{n-1}^{\dagger}\right) I$ be harmonic and belong to Ker $\widetilde{\partial_{\underline{z}}^{\dagger}}$, i.e. $\widetilde{\partial_{\underline{z}}^{\dagger}} g^{1}=0$ and $\widetilde{\partial_{\underline{z}}^{\dagger}} \widetilde{\partial}_{\underline{z}} g^{1}+\partial_{z_{n}} \partial_{\bar{z}_{n}} g^{1}=0$. Let $G^{0}$ be a solution of $\partial_{z_{n}} g^{0}=\widetilde{\partial}_{\underline{z}} g^{1}$ and put

$$
g^{0}=G^{0}+h^{0}\left(\bar{z},, \underline{\widetilde{z}}, \underline{z}^{\dagger}\right)
$$

where $h^{0} \in K e r \widetilde{\partial}_{\underline{z}}$ satisfies

$$
\widetilde{\partial_{\underline{z}}^{\dagger}} h^{0}=-\partial_{\bar{z}_{n}} g^{1}-\widetilde{\partial_{\underline{z}}^{\dagger}} G^{0}
$$

Then the function $g^{0}$ enjoys the following properties in $\Omega$ :

(i) $\widetilde{\partial_{\underline{z}}} g^{0}=0, \widetilde{\partial_{\underline{z}}^{\dagger}} g^{0}=-\partial_{\bar{z}_{n}} g^{1}, \partial_{z_{n}} g^{0}=\widetilde{\partial}_{\underline{z}} g^{1}$;

(ii) $g^{0}$ is harmonic;

(iii) $g^{0}+\mathfrak{f}_{n}^{\dagger} g^{1}$ is Hermitean monogenic.

Also here we may give an illustrative example in $\mathbb{R}^{6} \simeq \mathbb{C}^{3}$, now starting from the function $g^{1}=\frac{1}{2} \bar{z}_{3} z_{2}^{2} I$, which takes values in $\bigwedge\left(\mathfrak{f}_{1}^{\dagger}, \mathfrak{f}_{2}^{\dagger}\right) I$ and belongs to $\operatorname{Ker} \widetilde{\partial_{\underline{z}}^{\dagger}} \cap \operatorname{Ker} \Delta$. The equation to be solved reads $\partial_{z_{3}} g^{0}=\bar{z}_{3} z_{2} \mathfrak{f}_{2}^{\dagger} I$, with solution $G^{0}=z_{3} \bar{z}_{3} z_{2} f_{2}^{\dagger} I$, from which we obtain that

$$
g^{0}=z_{3} \bar{z}_{3} z_{2} \mathrm{f}_{2}^{\dagger} I+h^{0}\left(\bar{z}_{3}, z_{1}, \bar{z}_{1}, z_{2}, \bar{z}_{2}\right)
$$

This function $h^{0}$ is subject to the conditions $\widetilde{\partial_{\underline{z}}} h^{0}=0$ and $\widetilde{\partial_{\underline{z}}^{\dagger}} h^{0}=-\frac{1}{2} z_{2}^{2} I$. The function

$$
h^{0}=-\frac{1}{8} z_{2}^{2} \bar{z}_{1} \mathfrak{f}_{1}^{\dagger} I-\frac{3}{8} \bar{z}_{2} z_{2}^{2} \mathfrak{f}_{2}^{\dagger} I-\frac{1}{4} z_{2} z_{1} \bar{z}_{1} \mathfrak{f}_{2}^{\dagger} I
$$

satisfies the above equations, whence the function $g^{0}$ takes the form

$$
g^{0}=z_{3} \bar{z}_{3} z_{2} \mathrm{f}_{2}^{\dagger} I-\frac{1}{8} z_{2}^{2} \bar{z}_{1} \mathfrak{f}_{1}^{\dagger} I-\frac{3}{8} \bar{z}_{2} z_{2}^{2} \mathrm{f}_{2}^{\dagger} I-\frac{1}{4} z_{2} z_{1} \bar{z}_{1} \mathrm{f}_{2}^{\dagger} I
$$

The resulting function $g^{0}+\mathfrak{f}_{3}^{\dagger} g^{1}$ may then indeed be verified to be Hermitean monogenic in $\mathbb{C}^{3}$. 


\section{Complex differential forms}

In the previous section we have seen that the construction of a Hermitean conjugate pair in an open connected region $\Omega \subset \mathbb{C}^{n}$ requires solving the equation $\widetilde{\partial}_{\underline{z}} h=f$, where $f$ is in $\operatorname{Ker} \widetilde{\partial}_{\underline{z}} \cap \operatorname{Ker} \Delta$. This problem has the nature of a "Poincaré lemma" for the operator $\widetilde{\partial}_{\underline{z}}$ and the aim of this section is precisely to treat this Poincaré lemma for the Hermitean Dirac operators acting on functions with values in spinor space. To that end we will fully exploit the identification of the Hermitean Clifford analysis setting with the one of complex differential forms, as described in detail in [14]. More precisely we will translate our Poincaré lemma to the well-known $\bar{\partial}$-problem for complex differential forms.

In an open connected region $\Omega$ of $\mathbb{C}^{n} \simeq \mathbb{R}^{2 n}$ we consider the space $\bigwedge^{p, q}(\Omega)$ of complex differential forms of bidegree $(p, q)$ :

$$
\omega^{p, q}=\sum_{|J|=p} \sum_{|K|=q} \omega_{J, K}\left(\underline{z}, \underline{z}^{\dagger}\right) d z_{J} \wedge d \bar{z}_{K}
$$

where $\omega_{J, K}\left(z_{1}, \ldots, z_{n}, \bar{z}_{1}, \ldots, \bar{z}_{n}\right)$ are scalar-valued smooth functions in $\Omega$ and

$$
\begin{aligned}
d z_{J} & =d z_{j_{1}} \wedge \ldots \wedge d z_{j_{p}}, & & j_{1}<j_{2}<\ldots<j_{p} \\
d \overline{z_{K}} & =d \bar{z}_{k_{1}} \wedge \ldots \wedge d \bar{z}_{k_{q}}, & & k_{1}<k_{2}<\ldots<k_{q}
\end{aligned}
$$

The traditional complex derivatives in this setting are $\partial, \bar{\partial}, \partial^{*}$ and $\bar{\partial}^{*}$, given by

$$
\begin{aligned}
\partial & =\sum_{i=1}^{n} \partial_{z_{i}} d z_{i} \wedge: \bigwedge^{p, q} \longrightarrow \bigwedge^{p+1, q} \\
\bar{\partial} & =\sum_{i=1}^{n} \partial_{\bar{z}_{i}} d \bar{z}_{i} \wedge: \bigwedge^{p, q} \longrightarrow \bigwedge^{p, q+1} \\
\partial^{*} & \left.=\sum_{i=1}^{n} \partial_{\bar{z}_{i}} d \bar{z}_{i} \bullet=\sum_{i=1}^{n} \partial_{\bar{z}_{i}}\left(-2 \partial_{z_{i}}\right\rfloor\right): \bigwedge^{p, q} \longrightarrow \bigwedge^{p-1, q} \\
\bar{\partial}^{*} & \left.=\sum_{i=1}^{n} \partial_{z_{i}} d z_{i} \bullet=\sum_{i=1}^{n} \partial_{z_{i}}\left(-2 \partial_{\bar{z}_{i}}\right\rfloor\right): \bigwedge^{p, q} \longrightarrow \bigwedge^{p, q-1}
\end{aligned}
$$

where $d z_{i} \bullet$ and $d \bar{z}_{i} \bullet$ are alternative notations for the contraction operators, inspired by the dotproduct of the Clifford algebra. The complex derivatives satisfy the following Kähler identities.

Property 1. For the complex derivatives acting on complex differential forms one has

(i) $\partial^{2}=\bar{\partial}^{2}=\partial^{* 2}=\bar{\partial}^{* 2}=0$;

(ii) $\partial \bar{\partial}^{*}+\bar{\partial}^{*} \partial=0=\partial^{*} \bar{\partial}+\bar{\partial} \partial^{*}$;

(iii) $\partial \bar{\partial}+\bar{\partial} \partial=0=\partial^{*} \bar{\partial}^{*}+\bar{\partial}^{*} \partial^{*}$;

(iv) $\partial \partial^{*}+\partial^{*} \partial=-\frac{1}{2} \Delta=\overline{\partial \partial}^{*}+\bar{\partial}^{*} \bar{\partial}$.

In several complex variables theory the following theorem is of course well-known; it solves the famous $\bar{\partial}$-problem.

Theorem 5. If $\Omega \subset \mathbb{C}^{n}$ is pseudoconvex and $\varphi$ is a $C_{\infty}$-smooth $(p, q+1)$-form on $\Omega$, satisfying $\bar{\partial} \varphi=0$, then there exists a $C_{\infty}$-smooth $(p, q)$-form $\psi$ on $\Omega$, such that $\bar{\partial} \psi=\varphi$.

As in the one complex variable case (see Lemma 3), we can the solutions of the $\bar{\partial}$-problem may be represented by an integral formula. Indeed, we have the following result, involving the Koppelman-Leray integral transform $R_{\Omega}$. 
Lemma 4 (see [24, 3.2.3 and 2.1.6]). Let $\Omega \subset \mathbb{C}^{n}$ be a strictly pseudoconvex open set and let $\varphi$ be a bounded $C_{\infty}$-smooth $(0, q+1)$-form on $\Omega$, satisfying $\bar{\partial} \varphi=0$. Then $\psi:=R_{\Omega} \varphi$ is a bounded $C_{\infty}$-smooth solution of $\bar{\partial} \psi=\varphi$ on $\Omega$.

Similarly one can solve the $\partial$-problem, using the corresponding Koppelman-Leray integral transform $\widetilde{R}_{\Omega}$.

Theorem 4'. If $\Omega \subset \mathbb{C}^{n}$ is pseudoconvex and $\varphi$ is a $C_{\infty}$-smooth $(p+1, q)$-form on $\Omega$, satisfying $\partial \varphi=0$, then there exists a $C_{\infty}$-smooth $(p, q)$-form $\psi$ on $\Omega$, such that $\partial \psi=\varphi$.

Lemma 2'. Let $\Omega \subset \mathbb{C}^{n}$ be a strictly pseudoconvex open set and let $\varphi$ be a bounded $C_{\infty}$-smooth $(p+1,0)$-form on $\Omega$, satisfying $\partial \varphi=0$. Then $\psi:=\widetilde{R}_{\Omega} \varphi$ is a bounded $C_{\infty}$-smooth solution of $\partial \psi=\varphi$ on $\Omega$.

Both theorems may be refined by taking into account the adjoint complex derivatives $\partial^{*}$ and $\bar{\partial}^{*}$. We will prove the second one.

Theorem 5. If $\Omega \subset \mathbb{C}^{n}$ is pseudoconvex and $\varphi$ is a $C_{\infty}$-smooth $(p, q+1)$-form on $\Omega$, satisfying $\bar{\partial} \varphi=0$, then there exists a $C_{\infty}$-smooth $(p, q)$-form $\psi$ on $\Omega$, such that

(i) $\bar{\partial} \psi=\varphi$;

(ii) $\bar{\partial}^{*} \psi=0$,

or in other words: $\operatorname{Ker}^{(p, q+1)} \bar{\partial}=\bar{\partial}\left(\operatorname{Ker}^{(p, q)} \bar{\partial}^{*}\right)$.

Theorem 5'. If $\Omega \subset \mathbb{C}^{n}$ is pseudoconvex and $\varphi$ is a $C_{\infty}$-smooth $(p+1, q)$-form on $\Omega$, satisfying $\partial \varphi=0$, then there exists a $C_{\infty}$-smooth $(p, q)$-form $\psi$ on $\Omega$, such that

(i) $\partial \psi=\varphi$;

(ii) $\partial^{*} \psi=0$,

or in other words: $\operatorname{Ker}^{(p+1, q)} \partial=\partial\left(\operatorname{Ker}^{(p, q)} \partial^{*}\right)$.

\section{Proof}

From Theorem 4' we already know that, given $\partial \varphi^{(p+1, q)}=0$, there exists a form $\alpha^{(p, q)}$ such that in $\Omega$ there holds $\partial \alpha^{(p, q)}=\varphi^{(p+1, q)}$. It is also known that $\left(-\frac{1}{2} \Delta\right)=\partial \partial^{*}+\partial^{*} \partial: \bigwedge^{p, q} \longrightarrow \bigwedge^{p, q}$ is surjective, so there ought to exist a form $\beta^{(p, q)}$ such that

$$
\left(-\frac{1}{2} \Delta\right) \beta^{(p, q)}=\partial \partial^{*} \beta^{(p, q)}+\partial^{*} \partial \beta^{(p, q)}=-\alpha^{(p, q)}
$$

Put $\psi^{(p, q)}=\alpha^{(p, q)}+\partial \partial^{*} \beta^{(p, q)}$. Then, as $\partial^{2}=\partial^{* 2}=0$, we obtain

(i) $\partial \psi^{(p, q)}=\partial \alpha^{(p, q)}=\varphi^{(p+1, q)}$;

(ii) $\partial^{*} \psi^{(p, q)}=\partial^{*} \alpha^{(p, q)}+\partial^{*} \partial \partial^{*} \beta^{(p, q)}=\partial^{*} \alpha^{(p, q)}+\partial^{*}\left(-\frac{1}{2} \Delta\right) \beta^{(p, q)}=\partial^{*} \alpha^{(p, q)}-\partial^{*} \alpha^{(p, q)}=0$

Using the Hodge "star"-operator $*: \bigwedge^{p, q} \longrightarrow \bigwedge^{n-p, n-q}$ for which $(*)^{2}=(-1)^{(p+q)^{2}}$ and

$$
\bar{\partial}^{*}=* \partial *, \quad \partial=* \bar{\partial}^{*} *, \quad \partial^{*}=* \bar{\partial} *, \quad \bar{\partial}=* \partial^{*} *
$$

the following dual theorems are easily obtained.

Theorem 6. If $\Omega \subset \mathbb{C}^{n}$ is pseudoconvex and $\varphi$ is a $C_{\infty}$-smooth $(p, q-1)$-form on $\Omega$ satisfying $\bar{\partial}^{*} \varphi=0$, then there exists a $C_{\infty}$-smooth $(p, q)$-form $\psi$ on $\Omega$ such that 
(i) $\bar{\partial}^{*} \psi=\varphi$;

(ii) $\bar{\partial} \psi=0$,

or in other words: $\operatorname{Ker}^{(p, q-1)} \bar{\partial}^{*}=\bar{\partial}^{*}\left(\operatorname{Ker}^{(p, q)} \bar{\partial}\right)$.

Theorem 6'. If $\Omega \subset \mathbb{C}^{n}$ is pseudoconvex and $\varphi$ is a $C_{\infty}$-smooth $(p-1, q)$-form on $\Omega$ satisfying $\partial^{*} \varphi=0$, then there exists a $C_{\infty}$-smooth $(p, q)$-form $\psi$ on $\Omega$ such that

(i) $\partial^{*} \psi=\varphi$;

(ii) $\partial \psi=0$,

or in other words: $\operatorname{Ker}^{(p-1, q)} \partial^{*}=\partial^{*}\left(\operatorname{Ker}^{(p, q)} \partial\right)$.

Of course, we can again represent the solutions provided by the Theorems 5, 5', 6 and 6 ' by the corresponding integral formulas. For example, we have the following result.

Lemma 3. Let $\Omega \subset \mathbb{C}^{n}$ be a strictly pseudoconvex open set and let $\varphi$ be a bounded $C_{\infty}$-smooth $(p+1,0)$-form on $\Omega$, satisfying $\partial \varphi=0$. Put

$$
\psi:=\widetilde{R}_{\Omega} \varphi+2 \partial \partial^{*} U_{\Omega} \widetilde{R}_{\Omega} \varphi .
$$

Here $\widetilde{R}_{\Omega}$ is the integral transform from Lemma 2' and $U_{\Omega}$ is the Newtonian potential, that is,

$$
U_{\Omega} \alpha(\underline{X})=\int_{\Omega} \Gamma(\underline{X}-\underline{Y}) \alpha(\underline{Y}) d \underline{Y}, \underline{X} \in \mathbb{R}^{2 n}
$$

with $\Gamma$ the fundamental solution of the Laplace operator $\Delta$.

Then $\psi$ is a $C_{\infty}$-smooth $(p, 0)$-form on $\Omega$, such that $\partial \psi=\varphi$ and $\partial^{*} \psi=0$.

Proof

We can follow the proof of Theorem 5' and use Lemma 2' and the well-known fact that, for each bounded $C_{\infty}$-smooth function $\alpha$ on a bounded set $\Omega \subset \mathbb{R}^{2 n}$, the function $\beta:=U_{\Omega} \alpha$ is a $C_{\infty}$-smooth solution of the Poisson equation $\Delta \beta=\alpha$ on $\Omega$.

In the sequel we will use Theorems 5' and 6' for $(r, 0)$-forms.

In [14] it was shown in detail how the world of complex differential forms in an open connected region $\Omega$ of $\mathbb{C}^{n}$ and the world of Clifford algebra valed multivector functions in $\Omega$ may be identified in a natural way. The fundamental identifications read:

$$
\begin{aligned}
& \mathfrak{f}_{j}^{\dagger} \wedge \longleftrightarrow-\frac{1}{2} d z_{j} \wedge \\
& \mathfrak{f}_{j} \wedge \longleftrightarrow \frac{1}{2} d \bar{z}_{j} \wedge
\end{aligned}
$$

$$
\begin{aligned}
& \left.\mathfrak{f}_{j}^{\dagger} \bullet-\frac{1}{2} d z_{j} \bullet=\partial_{\bar{z}_{j}}\right\rfloor \\
& \left.\mathfrak{f}_{j} \bullet \longleftrightarrow \frac{1}{2} d \bar{z}_{j} \bullet=-\partial_{z_{j}}\right\rfloor
\end{aligned}
$$

and

$$
\begin{array}{llrl}
\partial_{\underline{z}}^{\dagger} \wedge & \frac{1}{2} \bar{\partial} & \partial_{\underline{\underline{t}}}^{\dagger} \bullet & \frac{1}{2} \partial^{*} \\
\partial_{\underline{z}} \wedge \longleftrightarrow-\frac{1}{2} \partial & \partial_{\underline{z}} \bullet \longleftrightarrow-\frac{1}{2} \bar{\partial}^{*}
\end{array}
$$

Now consider a smooth function $F: \Omega \subset \mathbb{C}^{n} \longrightarrow \mathbb{S}_{n}^{(r)}=\bigwedge^{(r)}\left(\mathfrak{f}_{1}^{\dagger}, \ldots, \mathfrak{f}_{n}^{\dagger}\right) I$ of the form

$$
F=\varphi\left(\underline{z}, \underline{z}^{\dagger}\right) \mathfrak{f}_{i_{1}}^{\dagger} \wedge \ldots \wedge \mathfrak{f}_{i_{r}}^{\dagger} I=\varphi\left(\underline{z}, \underline{z}^{\dagger}\right) \omega^{(r)} I
$$

where $\varphi\left(\underline{z}, \underline{z}^{\dagger}\right)$ is scalar-valued, and associate to it, respecting the above identification rules, the complex $(r, 0)$-form

$$
\widetilde{F}=\varphi\left(\underline{z}, \underline{z}^{\dagger}\right)\left(-\frac{1}{2} d z_{i_{1}}\right) \wedge \ldots \wedge\left(-\frac{1}{2} d z_{i_{r}}\right)=\varphi\left(\underline{z}, \underline{z}^{\dagger}\right) \widetilde{\omega}^{(r)}
$$


where we have put

$$
\widetilde{\omega}^{(r)}=\left(-\frac{1}{2}\right)^{r} d z_{i_{1}} \wedge \ldots \wedge d z_{i_{r}}
$$

We want to see how the action of the Hermitean Dirac operators $\partial_{\underline{z}}$ and $\partial_{\underline{z}}^{\dagger}$ on $F$ translate into actions on the corresponding complex differential form $\widetilde{F}$. To that end we calculate

$$
\partial_{\underline{z}} F=\sum_{j=1}^{n} \partial_{z_{j}} \varphi \mathfrak{f}_{j}^{\dagger}\left(\mathfrak{f}_{i_{1}}^{\dagger} \wedge \ldots \wedge \mathfrak{f}_{i_{r}}^{\dagger}\right) I=\sum_{j=1}^{n} \partial_{z_{j}} \varphi \mathfrak{f}_{j}^{\dagger} \mathfrak{f}_{i_{1}}^{\dagger} \ldots \mathfrak{f}_{i_{r}}^{\dagger} I=\sum_{j=1}^{n} \partial_{z_{j}} \varphi \omega_{j}^{(r+1)} I
$$

with

and

$$
\omega_{j}^{(r+1)}= \begin{cases}0 & j \in\left\{i_{1}, \ldots, i_{r}\right\} \\ \mathfrak{f}_{j}^{\dagger} \wedge \mathfrak{f}_{i_{1}}^{\dagger} \wedge \ldots \wedge \mathfrak{f}_{i_{r}}^{\dagger} & j \notin\left\{i_{1}, \ldots, i_{r}\right\}\end{cases}
$$

$$
\partial_{\underline{z}}^{\dagger} F=\sum_{j=1}^{n} \partial_{\bar{z}_{j}} \varphi \mathfrak{f}_{j}\left(\mathfrak{f}_{i_{1}}^{\dagger} \wedge \ldots \wedge \mathfrak{f}_{i_{r}}^{\dagger}\right) I=\sum_{j=1}^{n} \partial_{\bar{z}_{j}} \varphi \mathfrak{f}_{j} \mathfrak{f}_{i_{1}}^{\dagger} \ldots \mathfrak{f}_{i_{r}}^{\dagger} I=\sum_{j=1}^{n} \partial_{\bar{z}_{j}} \varphi \omega_{j}^{(r-1)} I
$$

with

$$
\omega_{j}^{(r-1)}= \begin{cases}0 & j \notin\left\{i_{1}, \ldots, i_{r}\right\} \\ (-1)^{k-1} \mathfrak{f}_{i_{1}}^{\dagger} \wedge \ldots \wedge\left[\mathfrak{f}_{i_{k}}^{\dagger}\right] \wedge \ldots \wedge \mathfrak{f}_{i_{r}}^{\dagger} \quad j=i_{k}, k \in\{1, \ldots, r\}\end{cases}
$$

The complex differential forms associated to $\partial_{\underline{z}} F$ and $\partial_{\underline{z}}^{\dagger} F$ then are given by

$$
\widetilde{\partial_{\underline{z}} F}=\sum_{j=1}^{n} \partial_{z_{j}} \varphi \widetilde{\omega}_{j}^{(r+1)}
$$

with

$$
\widetilde{\omega}_{j}^{(r+1)}= \begin{cases}0 & j \in\left\{i_{1}, \ldots, i_{r}\right\} \\ \left(-\frac{1}{2}\right)^{r+1} d z_{j} \wedge d z_{i_{1}} \wedge \ldots \wedge d z_{i_{r}} & j \notin\left\{i_{1}, \ldots, i_{r}\right\}\end{cases}
$$

and

$$
\widetilde{\partial_{\underline{z}}^{\dagger} F}=\sum_{j=1}^{n} \partial_{\bar{z}_{j}} \varphi \widetilde{\omega}_{j}^{(r-1)}
$$

with

$$
\widetilde{\omega}_{j}^{(r-1)}= \begin{cases}0 & j \notin\left\{i_{1}, \ldots, i_{r}\right\} \\ (-1)^{k-1}\left(-\frac{1}{2}\right)^{r-1} d z_{i_{1}} \wedge \ldots \wedge\left[d z_{i_{k}}\right] \wedge \ldots \wedge d z_{i_{r}} & j=i_{k}, k \in\{1, \ldots, r\}\end{cases}
$$

On the other hand we compute the action of $\partial$ and $\partial^{*}$ on the form $\widetilde{F}$, obtaining

$$
\partial \widetilde{F}=\sum_{j=1}^{n} \partial_{z_{j}} \varphi\left(\underline{z}, \underline{z}^{\dagger}\right) d z_{j} \wedge \widetilde{\omega}^{(r)}=\sum_{j=1}^{n} \partial_{z_{j}} \varphi\left(\underline{z}, \underline{z}^{\dagger}\right)(-2) \widetilde{\omega}_{j}^{(r+1)}
$$

and

$$
\begin{aligned}
\partial^{*} \widetilde{F} & \left.=\sum_{j=1}^{n} \partial_{z_{j}} \varphi\left(\underline{z}, \underline{z}^{\dagger}\right)\left(-2 \partial_{z_{j}}\right\rfloor\right) \widetilde{\omega}^{(r)} \\
& =\sum_{j=1}^{n} \partial_{z_{j}} \varphi\left(\underline{z}, \underline{z}^{\dagger}\right)(-2)\left(-\frac{1}{2}\right)^{r}(-1)^{k-1} d z_{i_{1}} \wedge \ldots \wedge\left[d z_{i_{k}}\right] \wedge \ldots \wedge d z_{i_{r}} \delta_{j, i_{k}} \\
& =\sum_{j=1}^{n} \partial_{z_{j}} \varphi\left(\underline{z}, \underline{z}^{\dagger}\right) \widetilde{\omega}_{j}^{(r-1)}
\end{aligned}
$$


and we finally find that

$$
\begin{aligned}
\widetilde{\partial_{\underline{z}} F} & =(-2) \partial \widetilde{F} \\
\partial_{\underline{z}}^{\dagger} F & =\partial^{*} \widetilde{F}
\end{aligned}
$$

As now $F=0$ iff $\widetilde{F}=0$ we readily obtain the theorems corresponding to Theorems 5 ' and 6 ', for multi-vector functions $F: \Omega \subset \mathbb{C}^{n} \longrightarrow \mathbb{S}_{n}^{(r)}$.

Theorem 7. If $\Omega \subset \mathbb{C}^{n}$ is pseudoconvex and $F: \Omega \longrightarrow \mathbb{S}_{n}^{(r)}$ is a $C_{\infty}$-smooth function satisfying $\partial_{\underline{z}} F=0$, then there exists a $C_{\infty}$-smooth function $G: \Omega \longrightarrow \mathbb{S}_{n}^{(r-1)}$ such that

(i) $\partial_{\underline{z}} G=F$;

(ii) $\partial_{\underline{z}}^{\dagger} G=0$.

Theorem 7'. If $\Omega \subset \mathbb{C}^{n}$ is pseudoconvex and $F: \Omega \longrightarrow \mathbb{S}_{n}^{(r)}$ is a $C_{\infty}$-smooth function satisfying $\partial_{\underline{z}}^{\dagger} F=0$, then there exists a $C_{\infty}$-smooth function $G: \Omega \longrightarrow \mathbb{S}_{n}^{(r+1)}$ such that

(i) $\partial_{\underline{z}}^{\dagger} G=F$;

(ii) $\partial_{z} G=0$.

The Theorems 5-7' may be seen as Poincaré lemmata for the operators involved. In view of the relations

$$
\bar{\partial}+\partial=d, \partial^{*}+\bar{\partial}^{*}=d^{*}, \underline{\partial}=2\left(\partial_{\underline{z}}^{\dagger}-\partial_{\underline{z}}\right)
$$

these results are in accordance with the traditional Poincaré lemmata for the differential operators $d, d^{*}$ and the Dirac operator $\underline{\partial}$ (see also $[5,6]$ ).

Remark 2. In case the given spinor-valued multi-vector function $F$ is a polynomial, we are able to construct explicitly the polynomials $G$ and $G^{\prime}$ where existence is guaranteed by the Theorems 7 and '7'. This is the topic of the next section.

As a corollary, by consecutively applying Theorems 7 and 7', we obtain for a given $C_{\infty}$ smooth multi-vector function $F_{0}^{(r)}: \Omega \subset \mathbb{C}^{n} \longrightarrow \mathbb{S}_{n}^{(r)}$, which is either in Ker $\partial_{\underline{z}}$ or in Ker $\partial_{\underline{z}}^{\dagger}$, a sequence of "primitives".

Corollary 1. If $\Omega$ is pseudoconvex and the $C_{\infty}$-smooth multi-vector function $F_{0}^{(r)}: \Omega \subset \mathbb{C}^{n} \longrightarrow$ $\mathbb{S}_{n}^{(r)}$ satisfies $\partial_{\underline{z}} F_{0}^{(r)}=0$ in $\Omega$, then there exists a sequence of multi-vector functions

$$
\left(F_{2 k-1}^{(r-1)}, F_{2 k}^{(r)}\right)_{k=1}^{\infty}
$$

such that

(i) $\partial_{\underline{z}} F_{2 k-1}^{(r-1)}=F_{2 k-2}^{(r)}, k=1,2, \ldots$;

(ii) $\partial_{\underline{z}}^{\dagger} F_{2 k-1}^{(r-1)}=0$;

(iii) $\partial_{\underline{z}} F_{2 k}^{(r)}=0$;

(iv) $\partial_{\underline{z}}^{\dagger} F_{2 k}^{(r)}=F_{2 k-1}^{(r-1)}$;

(v) $\left(\partial_{\underline{z}} \partial_{\underline{z}}^{\dagger}\right)^{k} F_{2 k}^{(r)}=F_{0}^{(r)}$;

(vi) $\partial_{\underline{z}}\left(\partial_{\underline{z}}^{\dagger} \partial_{\underline{z}}\right)^{k-1} F_{2 k-1}^{(r-1)}=F_{0}^{(r)}$. 
Corollary 2. If $\Omega$ is pseudoconvex and the $C_{\infty}$-smooth multi-vector function $G_{0}^{(r)}: \Omega \subset \mathbb{C}^{n} \longrightarrow$ $\mathbb{S}_{n}^{(r)}$ satisfies $\partial_{\underline{z}}^{\dagger} G_{0}^{(r)}=0$ in $\Omega$, then there exists a sequence of multi-vector functions

$$
\left(G_{2 k-1}^{(r+1)}, G_{2 k}^{(r)}\right)_{k=1}^{\infty}
$$

such that

(i) $\partial_{\underline{z}}^{\dagger} G_{2 k-1}^{(r+1)}=G_{2 k-2}^{(r)}, k=1,2, \ldots$;

(ii) $\partial_{\underline{z}} G_{2 k-1}^{(r+1)}=0$;

(iii) $\partial_{\underline{z}}^{\dagger} G_{2 k}^{(r)}=0$;

(iv) $\partial_{\underline{z}} G_{2 k}^{(r)}=G_{2 k-1}^{(r+1)}$;

(v) $\left(\partial_{\underline{z}}^{\dagger} \partial_{\underline{z}}\right)^{k} G_{2 k}^{(r)}=G_{0}^{(r)}$;

(vi) $\partial_{\underline{z}}^{\dagger}\left(\partial_{\underline{z}} \partial_{\underline{z}}^{\dagger}\right)^{k-1} G_{2 k-1}^{(r+1)}=G_{0}^{(r)}$.

Corollaries 1 and 2 can be summarized by the following schemes:

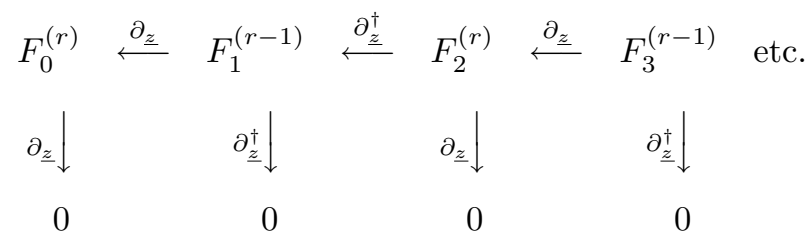

and

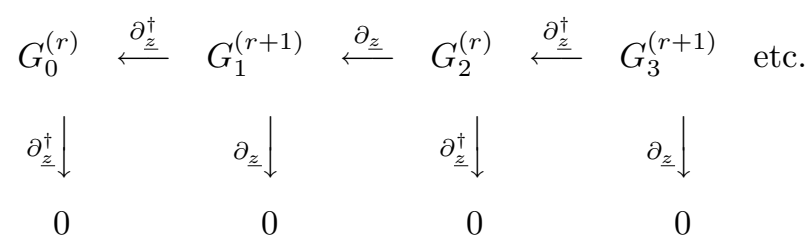

Corollary 3. If $\Omega \subset \mathbb{C}^{n}$ is pseudoconvex and $M_{0}^{(r)}: \Omega \longrightarrow \mathbb{S}_{n}^{(r)}$ is Hermitean monogenic in $\Omega$, then for each $k=1,2,3, \ldots$ there exists a $k$-harmonic function $H_{k}^{(r)} \in \operatorname{Ker} \Delta^{k}$ such that

$$
\left(\partial_{\underline{z}} \partial_{\underline{z}}^{\dagger}\right)^{k} H_{k}^{(r)}=M_{0}^{(r)}
$$

and

$$
\left(\partial_{\underline{z}}^{\dagger} \partial_{\underline{z}}\right)^{k} H_{k}^{(r)}=-M_{0}^{(r)}
$$

\section{Proof}

We know from Corollary 1 and 2 the existence of $F_{2 k}^{(r)}$ and $G_{2 k}^{(r)}$ such that

$$
\left(\partial_{\underline{z}} \partial_{\underline{z}}^{\dagger}\right)^{k} F_{2 k}^{(r)}=M_{0}^{(r)}, \quad \partial_{\underline{z}} F_{2 k}^{(r)}=0
$$

and

$$
\left(\partial_{\underline{z}}^{\dagger} \partial_{\underline{z}}\right)^{k}\left(-G_{2 k}^{(r)}\right)=-M_{0}^{(r)}, \quad \partial_{\underline{z}}^{\dagger} G_{2 k}^{(r)}=0
$$

Put $H_{k}^{(r)}=F_{2 k}^{(r)}-G_{2 k}^{(r)}$, then it follows that 
(i) $\left(\partial_{\underline{z}} \partial_{\underline{z}}^{\dagger}\right)^{k} H_{k}^{(r)}=\left(\partial_{\underline{z}} \partial_{\underline{z}}^{\dagger}\right)^{k} F_{2 k}^{(r)}=M_{0}^{(r)}$;

(ii) $\left(\partial_{\underline{z}}^{\dagger} \partial_{\underline{z}}\right)^{k} H_{k}^{(r)}=\left(\partial_{\underline{z}}^{\dagger} \partial_{\underline{z}}\right)^{k}\left(-G_{2 k}^{(r)}=-M_{0}^{(r)}\right.$;

(iii) $\Delta^{k} H_{k}^{(r)}=\left(\partial_{\underline{z}} \partial_{\underline{z}}^{\dagger}+\partial_{\underline{z}}^{\dagger} \partial_{\underline{z}}\right)^{k} H_{k}^{(r)}=\left(\left(\partial_{\underline{z}} \partial_{\underline{z}}^{\dagger}\right)^{k}+\left(\partial_{\underline{z}}^{\dagger} \partial_{\underline{z}}\right)^{k}\right) H_{k}^{(r)}=0$.

\section{The Poincaré lemmata for $\partial_{\underline{z}}$ and $\partial_{\underline{z}}^{\dagger}$ : the polynomial case}

In the foregoing section we have proven the solvability of the Poincaré lemma equations in the Hermitean Dirac operators $\partial_{\underline{z}}$ and $\partial_{\underline{z}}^{\dagger}$. In the case of polynomial data, where the geometric constraints are trivially fulfilled, we are able to construct explicitly the polynomial solution to these problems.

Let $\mathcal{P}_{a, b}^{(r)}$ be the space of homogeneous $\mathbb{S}_{n}^{(r)}$-valued polynomials of bidegree $(a, b)$, i.e. homogeneous of degree $a$ in the variables $\left(z_{1}, \ldots, z_{n}\right)$ and homogeneous of degree $b$ in the variables $\left(\bar{z}_{1}, \ldots, \bar{z}_{n}\right)$. We now prove in a constructive way the following polynomial version of Theorem 7 .

Theorem 8. If the polynomial $P_{a, b}^{(r)} \in \mathcal{P}_{a, b}^{(r)}$ satisfies $\partial_{\underline{z}} P_{a, b}^{(r)}=0$, then there exists $Q_{a+1, b}^{(r-1)} \in \mathcal{P}_{a+1, b}^{(r-1)}$ such that

(i) $\partial_{\underline{z}} Q_{a+1, b}^{(r-1)}=P_{a, b}^{(r)}$;

(ii) $\partial_{\underline{z}}^{\dagger} Q_{a+1, b}^{(r-1)}=0$.

The polynomial $Q_{a+1, b}^{(r-1)}$ is determined up to a Hermitean monogenic homogeneous polynomial in $\mathcal{P}_{a+1, b}^{(r-1)}$.

\section{Proof}

Invoking the Fischer decomposition for homogeneous polynomials in Ker $\partial_{\underline{z}}$ (see [9]), the homogeneous polynomial $P_{a, b}^{(r)} \in \operatorname{Ker} \partial_{\underline{z}}$ may be decomposed as follows:

$$
\begin{aligned}
P_{a, b}^{(r)}= & M_{a, b}^{(r)}+\underline{z}^{\dagger} M_{a, b-1}^{(r-1)}+|\underline{z}|^{2} \underline{z}^{\dagger} M_{a-1, b-2}^{(r-1)}+|\underline{z}|^{4} \underline{z}^{\dagger} M_{a-2, b-3}^{(r-1)}+\ldots \\
& +\left(\underline{z}^{\dagger} \underline{z}+\frac{a+r-1}{a+r} \underline{z} z^{\dagger}\right) M_{a-1, b-1}^{(r)}+|\underline{z}|^{2}\left(\underline{z^{\dagger}} \underline{z}+\frac{a+r-2}{a+r} \underline{z z}\right) M_{a-2, b-2}^{(r)}+\ldots
\end{aligned}
$$

where the $M_{\alpha, \beta}^{(s)}$ are Hermitean monogenic homogeneous polynomials in $\mathcal{P}_{\alpha, \beta}^{(s)}$. Now take $Q_{a+1, b}^{(r-1)} \in$ Ker $\partial_{z}^{\dagger}$ arbitrarily, then, in view of the Fischer decomposition for homogeneous polynomials in Ker $\partial_{\underline{z}}^{\dagger}$ (see also [9]), this polynomial may be decomposed as follows:

$$
\begin{aligned}
Q_{a+1, b}^{(r-1)}= & N_{a+1, b}^{(r-1)}+\underline{z} N_{a, b}^{(r)}+|\underline{z}|^{2} \underline{z} N_{a-1, b-1}^{(r)}+|\underline{z}|^{4} \underline{z} N_{a-2, b-2}^{(r)}+\ldots \\
& +\left(\underline{z z} \underline{z}^{\dagger}+\frac{b+n-r}{b+n-r+1} \underline{z}^{\dagger} \underline{z}\right) N_{a, b-1}^{(r-1)}+|\underline{z}|^{2}\left(\underline{z z} \underline{z}^{\dagger}+\frac{b+n-r-1}{b+n-r+1} \underline{z}^{\dagger} \underline{z}\right) N_{a-1, b-2}^{(r-1)}+\ldots
\end{aligned}
$$

where the $N_{\alpha, \beta}^{(s)}$ are Hermitean monogenic homogeneous polynomials in $\mathcal{P}_{\alpha, \beta}^{(s)}$. We will show that it is possible to find all the polynomials $N_{\alpha, \beta}^{(s)}$ in such a way that $\partial_{\underline{z}} Q_{a+1, b}^{(r-1)}=P_{a, b}^{(r)}$. This is done by directly computing $\partial_{\underline{z}} Q_{a+1, b}^{(r-1)}$ and idetifying the corresponding terms with those in $P_{a, b}^{(r)}$. As

$$
\partial_{\underline{z}}\left(|\underline{z}|^{2 j} \underline{z} N_{a-j, b-j}^{(r)}\right)=|\underline{z}|^{2(j-1)}\left((a+r) \underline{z} \underline{\dagger} \underline{z}+(a-j+r) \underline{z} \underline{z}^{\dagger}\right) N_{a-j, b-j}^{(r)}
$$


it follows that

$$
N_{a-j, b-j}^{(r)}=\frac{1}{a+r} M_{a-j, b-j}^{(r)}
$$

As

$$
\partial_{\underline{z}}\left(|\underline{z}|^{2 j}\left(\underline{z}^{\dagger}+\frac{b+n-r-j}{b+n-r+1} \underline{z}^{\dagger} \underline{z}\right) N_{a-j, b-j-1}^{(r-1)}\right)=\frac{(j+1)(a+b+n-j)}{b+n-r+1}|\underline{z}|^{2 j} \underline{z}^{\dagger} N_{a-j, b-j-1}^{(r-1)}
$$

it follows that

$$
N_{a-j, b-j-1}^{(r-1)}=\frac{b+n-r+1}{(j+1)(a+b+n-j)} M_{a-j, b-j-2}^{(r-1)}
$$

As $\partial_{\underline{z}} N_{a+1, b}^{(r-1)}=0$ trivially, the first term in the decomposition of the required conditions is completely determined up to an arbitrary Hermitean monogenic polynomial in $\mathcal{P}_{a+1, b}^{(r-1)}$.

In a similar way we can constructively prove the polynomial version of Theorem 7'.

Theorem 8'. If the polynomial $P_{a, b}^{(r)} \in \mathcal{P}_{a, b}^{(r)}$ satisfies $\partial_{\underline{z}}^{\dagger} P_{a, b}^{(r)}=0$, then there exists $Q_{a, b+1}^{(r+1)} \in \mathcal{P}_{a, b+1}^{(r+1)}$ such that

(i) $\partial_{\underline{z}}^{\dagger} Q_{a, b+1}^{(r+1)}=P_{a, b}^{(r)}$;

(ii) $\partial_{\underline{z}} Q_{a, b+1}^{(r+1)}=0$.

The polynomial $Q_{a, b+1}^{(r+1)}$ is determined up to a Hermitean monogenic homogeneous polynomial in $\mathcal{P}_{a, b+1}^{(r+1)}$

\section{The harmonic primitive or potential of a Hermitean mono- genic function}

As was pointed out in the introduction, the concept of a conjugate pair leads to a notion of potential or harmonic primitive of a holomorphic, repectively monogenic function. Also in the Hermitean monogenic setting it is possible to introduce this concept.

Assume that the open region $\Omega \subset \mathbb{C}^{n}$ and the function $g^{0}$ fulfill the requirements of Theorem 3 . Let, by Lemma 1, the function $F^{1}$ be a solution of the equation $\partial_{\bar{z}_{n}} F^{1}=-g^{0}$. This solution $F^{1}$ can always been chosen in Ker $\widetilde{\partial}_{\underline{z}}$. Indeed, if $\widetilde{\partial}_{\underline{z}} F^{1} \neq 0$, then $\widetilde{\partial}_{\underline{z}} F^{1} \in \operatorname{Ker} \widetilde{\partial}_{\underline{z}}$, and moreover it is independent of the variable $\bar{z}_{n}$ since $\partial_{\bar{z}_{n}} \widetilde{\partial}_{\underline{z}} F^{1}=-\widetilde{\partial}_{\underline{z}} g^{0}=0$. By Corollary 2 there exists a function $F^{2}\left(z_{n}, \underline{\widetilde{z}}, \widetilde{z}^{\dagger}\right) \in \operatorname{Ker} \widetilde{\partial}_{\underline{z}}$ such that $\widetilde{\partial_{\underline{z}}} \widetilde{\partial_{\underline{z}}^{\dagger}} F^{2}=\widetilde{\partial_{\underline{z}}} F^{1}$. The function $F^{1}-\widetilde{\partial_{\underline{z}}^{\dagger}} F^{2}$ clearly belongs to Ker $\widetilde{\partial}_{\underline{z}}$ and it is the desired solution: $\partial_{\bar{z}_{n}}\left(F^{1}-\widetilde{\partial_{\underline{z}}^{\dagger}} F^{2}\right)=-g^{0}-\partial_{\bar{z}_{n}} \widetilde{\partial_{\underline{z}}^{\dagger}} F^{2}=-g^{0}$ since the variable $\bar{z}_{n}$ does not occur in $F^{2}$. Now put $G^{1}=\widetilde{\partial_{\underline{z}}^{\dagger}} F^{1}$. Then $G^{1}$ is a solution of the equation $\partial_{\bar{z}_{n}} g^{1}=-\widetilde{\partial_{\underline{z}}^{\dagger}} g^{0}$ and the function $\partial_{z_{n}} g^{0}-\widetilde{\partial}_{z} G^{1}$ is independent of the variable $\bar{z}_{n}$. Again by Corollary 2 we know the existence of the function $W\left(z_{n}, \underline{\widetilde{z}}, \underline{\widetilde{z}}^{\dagger}\right) \in \operatorname{Ker} \widetilde{\partial_{\underline{z}}}$ such that $\widetilde{\partial_{\underline{z}}} \widetilde{\partial_{\underline{z}}^{\dagger}} W=\partial_{z_{n}} g^{0}-\widetilde{\partial_{\underline{z}}} G^{1}$. Put $h^{1}=\widetilde{\partial_{\underline{z}}^{\dagger}} W$, then $h^{1} \in \operatorname{Ker} \widetilde{\partial_{\underline{z}}^{\dagger}}$ and $\widetilde{\partial_{z}} h^{1}=\partial_{z_{n}} g^{0}-\widetilde{\partial}_{\underline{z}} G^{1}$, i.e. $h^{1}$ satisfies the conditions of Theorem 3. It follows that the conjugate component of $g^{0}$ is given by

$$
g^{1}=G^{1}+h^{1}=\widetilde{\partial_{\underline{z}}^{\dagger}}\left(F^{1}+W\right)=\widetilde{\partial_{\underline{z}}^{\dagger}} H^{1}
$$

where we have put

$$
H^{1}=F^{1}+W
$$

Note that the functions $W$ and $H^{1}$ have the same value space as the function $g^{0}$. 
Proposition 1. The function $H^{1}$, defined by (6.2), enjoys the following properties:

(a) $\widetilde{\partial_{\underline{z}}} \widetilde{\partial_{\underline{z}}^{\dagger}} H^{1}=\widetilde{\partial}_{\underline{z}} g^{1}=\partial_{z_{n}} g^{0}$;

(b) $\widetilde{\partial}_{\underline{z}} H^{1}=0$;

(c) $\partial_{\bar{z}_{n}} H^{1}=-g^{0}$;

(d) $\Delta H^{1}=0$.

\section{Proof}

(a) Take into account (6.1) and property (i) of Theorem 3.

(b) Follows from $F^{1} \in \operatorname{Ker} \widetilde{\partial}_{\underline{z}}$ and $W \in \operatorname{Ker} \widetilde{\partial}_{\underline{z}}$.

(c) Follows from $\partial_{\bar{z}_{n}} F^{1}=-\bar{g}^{0}$ and $\partial_{\bar{z}_{n}} W=0$ since the function $W$ does not contain this variable.

(d) We have that

$$
\begin{aligned}
\frac{1}{4} \Delta H^{1} & =\left(\partial_{\underline{z}} \partial_{\underline{z}}^{\dagger}+\partial_{\underline{z}}^{\dagger} \partial_{\underline{z}}\right) H^{1}=\left[\left(\widetilde{\partial_{\underline{z}}}+\mathfrak{f}_{n}^{\dagger} \partial_{z_{n}}\right)\left(\widetilde{\partial_{\underline{z}}^{\dagger}}+\mathfrak{f}_{n} \partial_{\bar{z}_{n}}\right)+\left(\widetilde{\partial_{\underline{z}}^{\dagger}}+\mathfrak{f}_{n} \partial_{\bar{z}_{n}}\right)\left(\widetilde{\partial}_{\underline{z}}+\mathfrak{f}_{n}^{\dagger} \partial_{z_{n}}\right)\right] H^{1} \\
& =\left(\widetilde{\partial_{\underline{z}}} \widetilde{\partial_{\underline{z}}^{\dagger}}+\widetilde{\partial_{\underline{f}}} \mathfrak{f}_{n} \partial_{\bar{z}_{n}}+\left(\mathfrak{f}_{n}^{\dagger} \mathfrak{f}_{n}+\mathfrak{f}_{n} \mathfrak{f}_{n}^{\dagger}\right) \partial_{z_{n}} \partial_{\bar{z}_{n}}\right) H^{1}=\partial_{z_{n}} g^{0}-\widetilde{\partial}_{\underline{z}} \mathfrak{f}_{n} g^{0}-\partial_{z_{n}} g^{0}=0
\end{aligned}
$$

By introducing the function $H^{1}$, the Hermitean monogenic function $g=g^{0}+\mathrm{f}_{n}^{\dagger} g^{1}$ corresponding to the conjugate pair $\left(g^{0}, g^{1}\right)$ now takes the form

$$
g=-\partial_{\bar{z}_{n}} H^{1}+\mathfrak{f}_{n}^{\dagger} \widetilde{\partial_{\underline{z}}^{\dagger}} H^{1}=-\mathfrak{f}_{n} \partial_{\bar{z}_{n}} \mathfrak{f}_{n}^{\dagger} H^{1}-\widetilde{\partial_{\underline{z}}^{\dagger}}{ }_{n}^{\dagger} H^{1}=-\left(\mathfrak{f}_{n} \partial_{\bar{z}_{n}}+\widetilde{\partial_{\underline{z}}^{\dagger}}\right)\left(\mathfrak{f}_{n}^{\dagger} H^{1}\right)=-\partial_{\underline{z}}^{\dagger}\left[f_{n}^{\dagger} H^{1}\right]
$$

which is very similar to the holomorphic and the monogenic cases, mentioned in the introduction. Whence we may call the function $H^{1}$ a harmonic potential or harmonic primitive of the first kind for the Hermitean monogenic function $g$. So we have proven the following theorem.

Theorem 9. Let $\Omega$ be open in $\mathbb{C}^{n}$ such that for each $\left(\underline{\underline{z}}_{*}, \underline{\widetilde{z}}_{*}^{\dagger}\right)$ fixed, the region $\Omega_{\left(\underline{\underline{z}}_{*}, \tilde{z}_{*}^{\dagger}\right)}=\left\{\left(\underline{z}, \underline{z}^{\dagger}\right) \in\right.$ $\Omega \mid\left(\underline{\widetilde{z}}, \underline{\underline{z}}^{\dagger}\right)=\left(\underline{\widetilde{z}}_{*}, \underline{\widetilde{z}}_{*}^{\dagger}\right)$ fixed $\}$ is simply connected, and that for each $\left(z_{n}^{*}, \bar{z}_{n}^{*}\right)$ fixed, the region $\Omega_{\left(z_{n}^{*}, \bar{z}_{n}^{*}\right)}=$ $\left\{\left(\underline{z}, \underline{z}^{\dagger}\right) \in \Omega \mid\left(z_{n}, \bar{z}_{n}\right)=\left(z_{n}^{*}, \bar{z}_{n}^{*}\right)\right.$ fixed $\}$ is pseudo-convex. Let the function $g^{0}: \Omega \rightarrow \Lambda^{\dagger}\left(\mathrm{f}_{1}^{\dagger}, \ldots, \mathrm{f}_{n-1}^{\dagger}\right) I$ be harmonic and belong to Ker $\widetilde{\partial}_{\underline{z}}$, i.e. $\widetilde{\partial}_{\underline{z}} g^{0}=0$ and $\widetilde{\partial_{\underline{z}}} \widetilde{\partial_{\underline{z}}^{\dagger}} g^{0}+\partial_{z_{n}} \partial_{\bar{z}_{n}} g^{0}=0$.

Then there exists a function $H^{1}: \Omega \longrightarrow \bigwedge^{\dagger}\left(\mathfrak{f}_{1}^{\dagger}, \ldots, \mathfrak{f}_{n-1}^{\dagger}\right) I$, called potential or primitive of the first kind, such that

(i) $H^{1}$ is harmonic in $\Omega$ and $H^{1} \in \operatorname{Ker} \widetilde{\partial_{z}}$;

(ii) $g^{1}=\widetilde{\partial_{\underline{z}}^{\dagger}} H^{1}$ is conjugate to $g^{0}$ in $\Omega$;

(iii) $g^{0}+\mathfrak{f}_{n}^{\dagger} g^{1}=-\partial_{\underline{z}}^{\dagger}\left(\mathfrak{f}_{n}^{\dagger} H^{1}\right)$ is Hermitean monogenic in $\Omega$.

This harmonic potential $H^{1}$ is given by

$$
H^{1}=F^{1}+W\left(z_{n}, \underline{\widetilde{z}}, \underline{\widetilde{z}}^{\dagger}\right)
$$

where $F^{1} \in \operatorname{Ker} \widetilde{\partial}_{\underline{z}}$ is a solution of $\partial_{\bar{z}_{n}} F^{1}=-g^{0}$ and where $W \in \operatorname{Ker} \widetilde{\partial}_{\underline{z}}$ is a solution of $\widetilde{\partial_{\underline{z}}} \widetilde{\partial_{\underline{z}}^{\dagger}} W=$ $\partial_{z_{n}} g^{0}-\widetilde{\partial_{\underline{z}}} \widetilde{\partial_{\underline{z}}^{\dagger}} F^{1}$.

In a similar way we can prove 
Theorem 9'. Let $\Omega$ be open in $\mathbb{C}^{n}$ such that for each $\left(\underline{\underline{z}}_{*}, \underline{\tilde{z}}_{*}^{\dagger}\right)$ fixed, the region $\Omega_{\left(\underline{\underline{z}}_{*}, \tilde{z}_{*}^{\dagger}\right)}=\left\{\left(\underline{z}, \underline{z}^{\dagger}\right) \in\right.$ $\Omega \mid\left(\underline{\underline{z}}, \underline{\underline{z}}^{\dagger}\right)=\left(\underline{\widetilde{z}}_{*}, \underline{\underline{z}}_{*}^{\dagger}\right)$ fixed $\}$ is simply connected, and that for each $\left(z_{n}^{*}, \bar{z}_{n}^{*}\right)$ fixed, the region $\Omega_{\left(z_{n}^{*}, \bar{z}_{n}^{*}\right)}=$ $\left\{\left(\underline{z}, \underline{z}^{\dagger}\right) \in \Omega \mid\left(z_{n}, \bar{z}_{n}\right)=\left(z_{n}^{*}, \bar{z}_{n}^{*}\right)\right.$ fixed $\}$ is pseudo-convex. Let the function $g^{1}: \Omega \rightarrow \Lambda^{\dagger}\left(\mathfrak{f}_{1}^{\dagger}, \ldots, \tilde{f}_{n-1}^{\dagger}\right) I$ be harmonic and belong to Ker $\widetilde{\partial_{\underline{z}}^{\dagger}}$, i.e. $\widetilde{\partial_{\underline{z}}^{\dagger}} g^{1}=0$ and $\widetilde{\partial_{\underline{z}}^{\dagger}} \widetilde{\partial_{\underline{z}}} g^{1}+\partial_{z_{n}} \partial_{\bar{z}_{n}} g^{1}=0$.

Then there exists a function $H^{0}: \Omega \longrightarrow \bigwedge^{\dagger}\left(\mathfrak{f}_{1}^{\dagger}, \ldots, \mathfrak{f}_{n-1}^{\dagger}\right) I$, called potential or primitive of the second kind, such that

(i) $H^{0}$ is harmonic in $\Omega$ and $H^{0} \in \operatorname{Ker} \widetilde{\partial_{\underline{z}}^{\dagger}}$;

(ii) $g^{0}=\widetilde{\partial}_{\underline{z}} H^{0}$ is conjugate to $g^{1}$ in $\Omega$;

(iii) $g^{0}+\mathfrak{f}_{n}^{\dagger} g^{1}=\partial_{\underline{z}} H^{0}$ is Hermitean monogenic in $\Omega$.

This harmonic potential $H^{0}$ is given by

$$
H^{0}=F^{0}+V\left(\bar{z}_{n}, \underline{\underline{z}}, \underline{\underline{z}}^{\dagger}\right)
$$

where $F^{0} \in \operatorname{Ker} \widetilde{\partial_{\underline{z}}^{\dagger}}$ is a solution of $\partial_{z_{n}} F^{0}=g^{1}$ and where $V \in \operatorname{Ker} \widetilde{\partial_{\underline{z}}^{\dagger}}$ is a solution of $\widetilde{\partial_{\underline{z}}^{\dagger}} \widetilde{\partial_{\underline{z}}} V=$ $\partial_{\bar{z}_{n}} g^{1}-\widetilde{\partial_{\underline{z}}^{\dagger}} \widetilde{\partial_{z}} F^{0}$.

Finally we illustrate these theorems by the example of Section 3. Starting with the conjugate pair $\left(g^{0}, g^{1}\right)=\left(\bar{z}_{2} z_{3}^{2} f_{1}^{\dagger} I, 2 z_{1} \bar{z}_{2} z_{3} I\right)$, the function $F^{1}=-\bar{z}_{2} z_{3}^{2} \bar{z}_{3} \mathrm{f}_{1}^{\dagger} I$ satisfies $\widetilde{\partial}_{\underline{z}} F^{1}=0$ and $\partial_{\bar{z}_{3}} F^{1}=-g^{0}$. Apparently $G^{1}=0$ and the function $W=2 z_{1} \bar{z}_{1} \bar{z}_{2} z_{3} \mathrm{f}_{1}^{\dagger} I$ satisfies the equation $\widetilde{\partial_{\underline{z}}} \widetilde{\partial_{\underline{z}}^{\dagger}} W=\partial_{z_{3}} g^{0}=$ $2 \bar{z}_{2} z_{3} \mathrm{f}_{1}^{\dagger} I$, the right-hand side not containing the variable $\bar{z}_{3}$. A harmonic potential $H^{1}$ of the first kind is then given by

$$
H^{1}=\left(-\bar{z}_{2} z_{3}^{2} \bar{z}_{3}+2 z_{1} \bar{z}_{1} \bar{z}_{2} z_{3}\right) \mathfrak{\dagger}_{1}^{\dagger} I
$$

and it is easily verified that it satisfies the following properties:

(a) $\widetilde{\partial_{\underline{z}}} \widetilde{\partial_{\underline{z}}^{\dagger}} H^{1}=\widetilde{\partial}_{\underline{z}}\left(2 z_{1} \bar{z}_{2} z_{3} I\right)=2 \bar{z}_{2} z_{3} \uparrow_{1}^{\dagger} I=\widetilde{\partial_{\underline{z}}} g^{1}=\partial_{z_{3}} g^{0}$

(b) $\widetilde{\partial}_{\underline{z}} H^{1}=0$;

(c) $\partial_{\bar{z}_{3}} H^{1}=-\bar{z}_{2} z_{3}^{2} \mathrm{f}_{1}^{\dagger} I=-g^{0}$;

(d) $\Delta H^{1}=0$;

(e) $-\partial_{\underline{z}}^{\dagger}\left[\mathrm{f}_{3}^{\dagger} H^{1}\right]=\bar{z}_{2} z_{3}^{2} \mathrm{f}_{1}^{\dagger} I+2 z_{1} \bar{z}_{2} z_{3} \mathrm{f}_{3}^{\dagger} I=g^{0}+\mathfrak{f}_{3}^{\dagger} g^{1}=g$;

(f) $H^{1}$ has the same value space as $g^{0}$.

As $\partial_{\bar{z}_{3}} g^{1}=0$ we may choose $V=0$, giving rise to the harmonic potential of the second kind $H^{0}=z_{1} \bar{z}_{2} z_{3}^{2} I$, for which it holds that

(a) $\widetilde{\partial_{\underline{z}}^{\dagger}} \widetilde{\partial_{\underline{z}}} H^{0}=\widetilde{\partial_{\underline{z}}^{\dagger}}\left(\bar{z}_{2} z_{3}^{2} \mathrm{f}_{1}^{\dagger} I\right)=0=\widetilde{\partial_{\underline{z}}^{\dagger}} g^{0}=-\partial_{\bar{z}_{3}} g^{1}$;

(b) $\widetilde{\partial_{\underline{z}}^{\dagger}} H^{0}=0$;

(c) $\partial_{z_{3}} H^{0}=2 z_{1} \bar{z}_{2} z_{3} I=g^{1}$;

(d) $\Delta H^{0}=0$;

(e) $\partial_{\underline{z}} H^{0}=\bar{z}_{2} z_{3}^{2} \mathrm{f}_{1}^{\dagger} I+2 z_{1} \bar{z}_{2} z_{3} \mathrm{f}_{3}^{\dagger} I=g^{0}+\mathrm{f}_{3}^{\dagger} g^{1}=g$;

(f) $H^{0}$ has the same value space as $g^{1}$. 


\section{Acknowledgements}

This research was carried out in the framework of a project supported by the Committee for Scientific Research (CWO) of the Faculty of Engineering and Architecture at Ghent University. Moreover, R. Lávička and V. Souček acknowledge support by the institutional grant MSM 0021620839 and by grant GA CR 201/08/0397.

\section{References}

[1] F. Brackx, J. Bureš, H. De Schepper, D. Eelbode, F. Sommen, V. Souček, Fundaments of Hermitean Clifford analysis - Part I: Complex structure, Compl. Anal. Oper. Theory 1 (3), 2007, 341-365.

[2] F. Brackx, J. Bureš, H. De Schepper, D. Eelbode, F. Sommen, V. Souček, Fundaments of Hermitean Clifford analysis - Part II: Splitting of $h$-monogenic equations, Complex Var. Elliptic Eq. 52 (10-11), 2007, 1063-1079.

[3] F. Brackx, B. De Knock, H. De Schepper, F. Sommen, On Cauchy and Martinelli-Bochner Formulae in Hermitean Clifford Analysis, Bull. Braz. Math. Soc. 40 (3), 2009, 395-416.

[4] F. Brackx, B. De Knock, H. De Schepper, A matrix Hilbert transform in Hermitean Clifford Analysis, J. Math. Anal. Appl. 344 (2), 2008, 1068-1078.

[5] F. Brackx, R. Delanghe, F. Sommen, Clifford Analysis, Pitman Publishers (Boston-LondonMelbourne, 1982).

[6] F. Brackx, R. Delanghe, F. Sommen, Differential forms and/or multivector functions, Cubo 7(2), 2005, 139-169.

[7] F. Brackx, H. De Schepper, N. De Schepper, F. Sommen, Hermitean Clifford-Hermite polynomials, Adv. Appl. Clifford Alg. 17 (3), 2007, 311-330.

[8] F. Brackx, H. De Schepper, D. Eelbode, V. Souček, The Howe Dual Pair in Hermitean Clifford Analysis, Rev. Mat. Iberoamericana 26 (2), 2010, 449-479.

[9] F. Brackx, H. De Schepper, R. Lávička, V. Souček, Fischer decompositions of kernels of Hermitean Dirac operators. In: T.E. Simos, G. Psihoyios, Ch. Tsitouras, Numerical Analysis and Applied Mathematics, AIP Conference Proceedings 1281, Rhodos, Greece (2010), 14841487.

[10] F. Brackx, H. De Schepper, R. Lávička, V. Souček, The Cauchy-Kovalevskaya Extension Theorem in Hermitean Clifford Analysis, J. Math. Anal. Appl. 381, 2011, 649-660.

[11] F. Brackx, H. De Schepper, F. Sommen, The Hermitian Clifford analysis toolbox, Adv. Appl. Cliff. Alg. 18 (3-4), 2008, 451-487.

[12] F. Brackx, H. De Schepper, F. Sommen, A Theoretical Framework for Wavelet Analysis in a Hermitean Clifford Setting, Communications on Pure and Applied Analysis 6 (3), 2007, $549-567$.

[13] F. Brackx, H. De Schepper, V. Souček, Fischer decompositions in Euclidean and Hermitean Clifford analysis, Archivum Mathematicum 46(5), 2010, 301-321. 
[14] F. Brackx, H. De Schepper, V. Souček, Differential forms versus multi-vector functions in Hermitean Clifford analysis, Cubo 13, 2011.

[15] R. B. Burckel, An Introduction to Classical Complex Analysis Vol. 1, Birkhäuser Verlag (Basel-Stuttgart, 1979).

[16] F. Colombo, I. Sabadini, F. Sommen, D. C. Struppa, Analysis of Dirac Systems and Computational Algebra, Birkhäuser (Boston, 2004).

[17] R. Delanghe, F. Sommen, V. Souček, Clifford algebra and spinor-valued functions - A function theory for the Dirac operator, Kluwer Academic Publishers (Dordrecht, 1992).

[18] D. Eelbode, Stirling numbers and Spin-Euler polynomials, Exp. Math. 16 (1), 2007, 55-66.

[19] D. Eelbode, Irreducible $\mathfrak{s l}(m)$-modules of Hermitean monogenics, Complex Var. Elliptic Equ. 53 (10), 2008, 975-987.

[20] D. Eelbode, Fu Li He, Taylor series in Hermitean Clifford Analysis, Complex Analysis and Operator Theory, DOI: 10.1007/s11785-009-0036-y.

[21] J. Gilbert, M. Murray, Clifford Algebra and Dirac Operators in Harmonic Analysis, Cambridge University Press (Cambridge, 1991).

[22] K. Gürlebeck, K. Habetha, W. Sprößig, Holomorphic functions in the plane and n-dimensional space. Translated from the 2006 German original. With 1 CD-ROM (Windows and UNIX), Birkhäuser Verlag (Basel, 2008).

[23] K. Gürlebeck, W. Sprößig, Quaternionic and Clifford Calculus for Physicists and Engineers, J. Wiley \& Sons (Chichester, 1997).

[24] G. M. Henkin, J. Leiterer, Theory of Functions on Complex Manifolds, Akademie-Verlag, Berlin, 1984.

[25] I. Porteous, Clifford Algebras and the Classical groups, Cambridge University Press (Cambridge, 1995).

[26] R. Rocha-Chavez, M. Shapiro, F. Sommen, Integral theorems for functions and differential forms in $\mathbb{C}_{m}$, Research Notes in Math. 428, Chapman\&Hall / CRC (New York, 2002).

[27] I. Sabadini, F. Sommen, Hermitian Clifford analysis and resolutions, Math. Meth. Appl. Sci. 25(16-18), 2002, 1395-1414.

[28] F. Sommen, D. Peña Peña, A Martinelli-Bochner formula for the Hermitian Dirac equation, Math. Meth. Appl. Sci. 30 (9), 2007, 1049-1055.

[29] E. Stein, G. Weiss, On the theory of harmonic functions of several variables, Part I: The theory of $H^{p}$ spaces, Acta Math. 103, 1960, 25-62. 